\section{To: (Receiving Organization) Distribution}

5. Proj./Prog./Dept./Div.:

Spent Nuclear Fuels Project

8. Originator Remarks:

For Release

11. Receiver Remarks:
3. From: (originating Organization) Spent Nuclear Fuel Evaluations

6. Cog. Engr.:

B. J. Makenas / $2 \mathrm{~T} 650$
Page 1 of 1. EDT $6 1 7 \longdiv { 4 4 4 }$
4. Related EDT No.:

$\mathrm{N} / \mathrm{A}$

7. Purchase Order No.: N/A

9. Equip./Component No.:

$N / A$

10. System/Bldg./Facility:

N/A $N / A$

13. Permit/Permit Application No.: N/A

14. Required Response Date: N/A

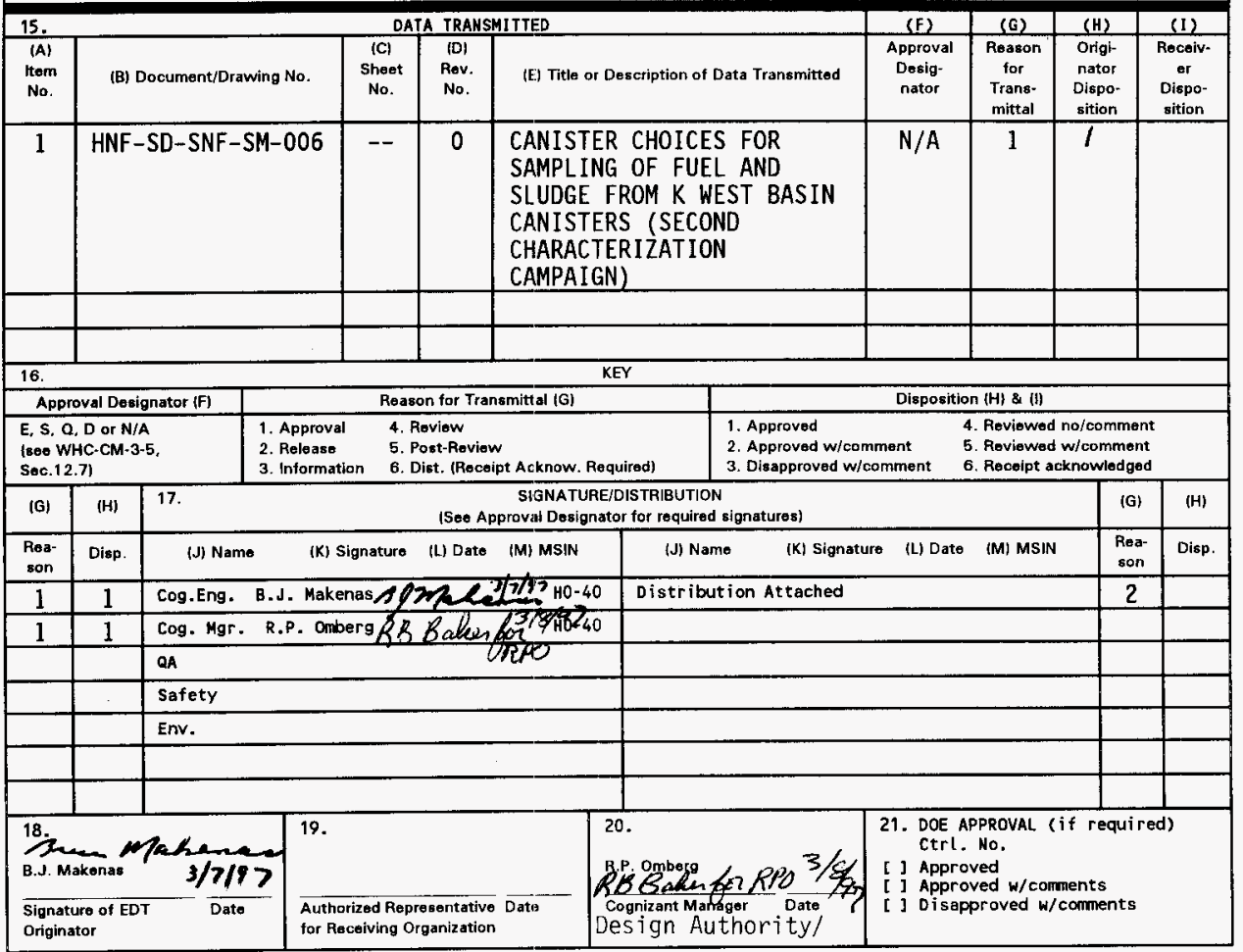




\section{CANISTER CHOICES FOR SAMPLING OF FUEL AND SLUDGE FROM $K$ WEST BASIN CANISTERS (SECOND CHARACTERIZATION CAMPAIGN)}

B. J. Makenas, D. J. Trimble, A. L. Pitner, R. B. Baker, and $L$. A. Lawrence

Duke Engineering and Services Hanford, Inc., Richland, WA 99352

U.S. Department of Energy Contract DE-AC06-96RL13200

EDT/ECN: $617444 \quad$ UC: 2070

Org Code: 2T650 Charge Code: LB026

B\&R Code: EW 7040000 Tata1 Pages: $46 \mathrm{cw}_{3-11-97}$

Key Words: K Basin, Sludge, N Reactor Fuel, Characterization

Abstract: The Characterization of Spent Nuclear Fuel in the sealed $K$ West canisters involves four steps, (1) sampling of gas/liquid, (2) "Lift-and-Look" visual examinations of fuel elements, (3) sampling of canister sludge, and (4) retrieving fuel for hot cell examinations. This document indicates the choices of particular canisters that were examined during the second characterization campaign in the Hanford $K$ West Basin.

TRADEMARK DISCLAIMER. Reference herein to any specific commercial product, process, or service by trade name, tradenark, manufacturer, or otherwise, does not necessarily constitute or imply its endorsement, recommendation; or favoring by the United States Government or any agency thereof or its contractors or subcontractors.

Printed in the United States of America. To obtain copies of this document, contact: Document Control Services, P.0. Box 950, Mailstop H6-08, Richland WA 99352, Phone (509) 372-2420; Fax (509) 376-4989.
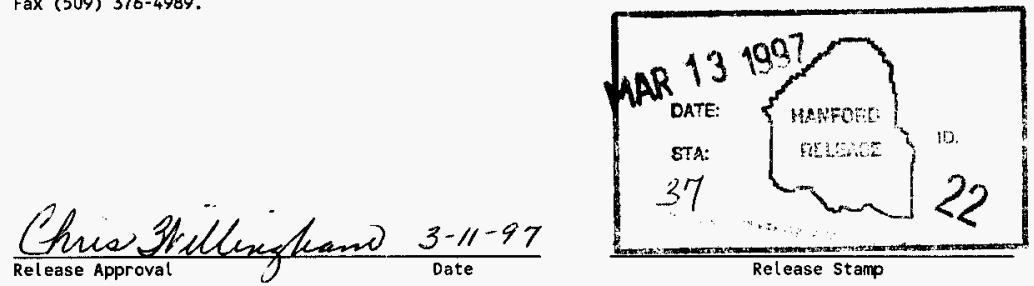

\section{Approved for Public Release}


HNF-SD-SNF-SM-006, Rev. 0

\section{CANISTER CHOICES FOR SAMPLING OF FUEL AND SLUDGE FROM $K$ WEST BASIN CANISTERS (SECOND CHARACTERIZATION CAMPAIGN)}

B. J. Makenas, D. J. Trimble, A. L. Pitner, R. B. Baker, and $L$. A. Lawrence

Duke Engineering and Services Hanford, Inc.

March 1997 
HNF-SD-SNF-SM-006, Rev. 0

\section{CONTENTS}

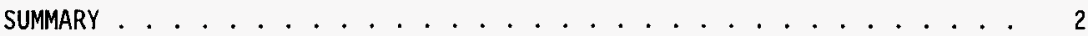

APPENDIX A RECOMMENDED CANISTERS FOR GAS AND LIQUID SAMPLING . . . 3

APPENDIX B RECOMMENDED CANISTERS FOR SLUDGE DEPTH MEASUREMENTS

AND VISUAL EXAMINATIONS . . . . . . . . . . . . . . 7

APPENDIX C RECOMMENDED CANISTERS FOR SLUDGE SAMPLING $\ldots \ldots \ldots$

APPENDIX D RECOMMENDED CANISTERS TO SUPPLY ELEMENTS

FOR HOT CELL EXAMINATIONS ............ 21 


\title{
CANISTER CHOICES FOR SAMPLING OF FUEL AND SLUDGE FROM $K$ WEST BASIN CANISTERS (SECOND CHARACTERIZATION CAMPAIGN)
}

\begin{abstract}
SUMMARY
The water-filled Hanford $K$ West Basin houses over 3,000 sealed canisters containing $N$ Reactor Spent Nuclear Fuel (SNF). During late CY 1996 and early 1997 a characterization campaign was conducted in the $K$ West Basin. It included the following steps:

A. Sampling of gas and liquid from approximately 50 sealed $K$ West canisters. Note: Each canister presents two barrels to be sampled.

B. Measurement of sludge depth in roughly 20 canisters using ultrasonic techniques concurrently with visual examination of selected elements from canisters.

C. Retrieval of sludge samples from nine canister barrels.

D. Retrieval of 15 fuel elements for shipment to hot cell examination facilities.

Steps $A, C$, and D, listed above, were planned and initiated through the issuance of formal Data Quality Objectives (DQO) documents. Specific canisters to be sampled or opened were specified through recommendation letters issued by the SNF Characterization group with concurrence from the $K$ Basin 0perations group. The purpose of this document is to collect these letters as evidence of the initial efforts to satisfy the goals set forth in the DQO process. Each of the following appendices, A through D, is a recommendation letter corresponding to the steps Tisted above. Applicable precursor $\mathrm{DQO}$ documents are referenced in each letter.
\end{abstract}




\section{HNF-SD-SNF-SM-006, Rev. 0}

A P P E N D I X A

\section{RECOMMENDED CANISTERS FOR GAS AND LIQUID SAMPLING}


HNF-SD-SNF-SM-006, Rev. 0

Westinghouse

Internal

Hanford Company

From: $\quad$ Spent Nuclear Fuel Evaluations

Phone: $\quad 376-5447$ HO-40

Date: $\quad$ August 7, 1996

Subject: CANISTER CHOICES FOR GAS/LIQUID SAMPLING

To:

R. P. Omberg

$\mathrm{HO}-40$

CC:

PZ 34
$H O-40$
$R 3-82$
$R 3-86$
$H 0-40$
$R 3-11$
$R 3-86$
$H O-40$
$X 3-85$
$H 0-40$
$P 7-34$

P. J. MacFarlan

$\mathrm{HO}-40$

R. Abrefah. PNNL

C. T. Miller

G. L. Miller

$\times 3-72$

G. Baston, MACTEC

K. R. Morris

T6-06

D. W. Bergmañn

K. L. Pearce

J. C. Fulton

J. Shuen, RL

$\times 3-67$

E. W. Gerber

D. W. Siddoway

R. A. Harris

J. A. Swenson

J. J. Jernberg

BJM $F$ ile/LB

L. A. Lawrence

DJT File/LB

R3-48

$57-41$

$\times 3-71$

R3-11

$\mathrm{HO}-40$

S. C. Marschman, PNNL P7-34

$\mathrm{HO}-4 \mathrm{O}$

Attached is a list of recommended canisters for the second gas/liquid sampling campaign in $K$ West Basin. The two tables presented in the attachment are for MK I and MK II canisters respectively. Included are 50 prime candidates plus eight alternates. Various candidates contain MK IA fuel, MK IV fuel, chips, D Grade fuel, known broken elements. water in-leakage (i.e., broken locking bars), and/or gas filled traps (suggesting corrosion). Based on a review of the current data base, an attempt was made to span a variety of encapsulation times and fuel burnups.

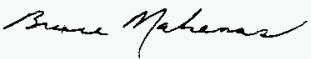

B. J. Makenas

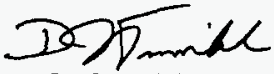

D. J. Trimble

$J \mathrm{mn}$

Attachment

CONCURRENCE:
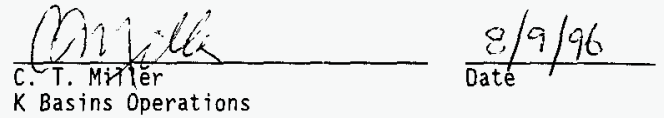

$B P^{2}$

Reference: B.J.Makenas,D.J.Trimble and D.1!. Berman, "Data Quality Objectives for Gas and

Liquid Samples from Sealed K lest Basin Canisters (second campaign), IHC-SD-SHF-DQ0-006, Rev.0.

August 1996. 


\begin{tabular}{|c|c|c|c|c|c|c|c|c|c|}
\hline & & & & & & & & & \\
\hline & Canister & & \multicolumn{2}{|c|}{ Euet } & No of & & & & \\
\hline Cube & S.N. & Key & Iype & modelsts & & BROKEN & \multicolumn{2}{|c|}{ ENCAPSULATION } & \\
\hline 0748 & 2600 & 13525 & MIA & & Assys & FUEL & DAIE & YEARS & $\%$ Pu240 \\
\hline $0754 *$ & 2775 & 13525 & MIA & At & 14 & & May-82 & 14.3 & 13.0 \\
\hline 1464 & 2554 & 12942 & $\overline{M I A}$ & $A M$ & 14 & & May-82 & 14.3 & 13.0 \\
\hline 1630 & 2660 & 13016 & MIA & $A M$ & 14 & & Apr-82 & 14.4 & 12.6 \\
\hline 2661 & 1730 & 12480 & MIA & Al & 14 & & Apr.82 & 14.4 & 12.8 \\
\hline $2804 \cdot$ & 1820 & 12480 & $\frac{\mathrm{MIA}}{\mathrm{M} \mid \mathrm{A}}$ & $\mathrm{AM}$ & 14 & & Aug-81 & 15.1 & 13.3 \\
\hline $2810^{*}$ & 1869 & 12480 & $\frac{\text { MIA }}{\text { MIA }}$ & $A M$ & 14 & & Aug-81 & 15.1 & 13.3 \\
\hline 2828 & $1556 \times$ & 12480 & MIA & AM & 14 & & Aug-81 & 15.1 & 13.3 \\
\hline 2913 & 161 & 10832 & $\frac{M I A}{M I A}$ & AM & 14 & & Aug-81 & 15.1 & 13.3 \\
\hline 2923 & 2667 & 13371 & $\frac{\text { MIA }}{\text { MIA }}$ & $\bar{A} \bar{A}$ & 14 & & Feb-81 & 15.6 & 12.5 \\
\hline $3617 \cdot$ & 1162 & 12127 & $-\frac{\mathrm{MIA}}{\mathrm{MIA}}$ & $A M$ & 14 & & Apr-82 & 14.4 & 12.5 \\
\hline 3619 & 1164 & 12127 & $\frac{1}{M I A}$ & AM & 14 & & Jul-81 & 15.2 & 13.7 \\
\hline 3839 & $1497 X$ & 11979 & & $\mathrm{AM}$ & 14 & & Jul-81 & 15.2 & 13.7 \\
\hline 4245 & 1226 & 11897 & $\frac{\text { MIA }}{\text { MIA }}$ & $\mathrm{AM}+\mathrm{AT}$ & $7+7$ & 1 outer & Jul-81 & 15.2 & 73.7 \\
\hline 4443 & $709 x$ & $11 \overline{806}$ & $\frac{\text { MIA }}{\text { MIA }}$ & $\mathrm{AF}+\mathrm{AM}$ & $3+11$ & 1 inner & Jun-81 & 15.3 & 13.8 \\
\hline 4459 & $102 x$ & 11806 & $\frac{\text { MIA }}{\text { M!A }}$ & $\mathrm{AM}$ & 14 & & Jun-81 & 15.3 & 13.3 \\
\hline 4463 & $200 x$ & 11806 & $\frac{M ! A}{M ! A}$ & $\mathrm{AM}$ & 14 & & Feb-81 & 15.6 & 13.3 \\
\hline $4 \overline{608}$ & 111 & 12852 & $\frac{\text { MIA }}{\text { MIV }}$ & $\mathrm{AM}$ & 14 & & Feb-81 & 15.6 & 13.3 \\
\hline 4612 & 316 & 12852 & $\frac{\text { MIV }}{\text { MIV }}$ & $\mathrm{BE}$ & 14 & & Mar-81 & 15.5 & 12.8 \\
\hline $4613^{*}$ & 315 & 12852 & $\frac{\text { MIV }}{\text { MIV }}$ & $\mathrm{BE}$ & 14 & & Mar-81 & 15.5 & 12.8 \\
\hline 4614 & 318 & 12852 & $\frac{\text { MIV }}{\text { MIV }}$ & $B E$ & 14 & & Mar-81 & 15.5 & 12.8 \\
\hline $5006^{\circ}$ & 760 & 13015 & $\frac{\text { MIV }}{\text { MIV }}$ & $\mathrm{BE}$ & 14 & & Mar-81 & 15.5 & 12.8 \\
\hline 5060 & 710 & 12942 & MIV & $\mathrm{BC}$ & 14 & & May-81 & 15.3 & 12.7 \\
\hline 5309 & 1619 & 13017 & MIV & $\mathrm{BE}$ & 14 & & May-81 & 15.3 & 12.3 \\
\hline 5366 & $943 X$ & 12852 & MIV & $\overline{B E}$ & 14 & pieces & Sep-81 & 15.0 & 11.0 \\
\hline 5369 & 1740 & 12852 & $\frac{\text { MIV }}{\text { MIV }}$ & $\mathrm{BE}$ & 14 & some & Sep-81 & 15.0 & 12.8 \\
\hline 5370 & 1560 & 12852 & MIV & $\mathrm{BE}$ & 14 & some & Sep-81 & 15.0 & 12.8 \\
\hline 5373 & 1860 & 13015 & MIV & $\mathrm{BE}$ & 14 & inners\&outers & Sep-81 & 15.0 & 12.8 \\
\hline 5753 & 9010 & 13372 & NAT & $7 E$ & 14 & inners\&outers & Sep- 81 & 15.0 & 11.8 \\
\hline 5850 & 9020 & 13524 & $\frac{\text { MIV }}{\text { MIV }}$ & BS & 14 & & Mar' 82 & 14.5 & 12.4 \\
\hline 5943 & 2800 & 13524 & MIV & $B A+B E+B S$ & $11+1+2$ & & Mar-82 & 14.5 & 12.5 \\
\hline & & & MIV & $\mathrm{BE}$ & 14 & & Mar-82 & 14.5 & 12.5 \\
\hline - = backup & candiate & & & & & & & & \\
\hline
\end{tabular}




\begin{tabular}{|c|c|c|c|c|c|c|c|c|c|c|c|c|c|}
\hline \multicolumn{14}{|c|}{ MARK II CANISTERS } \\
\hline & canistor & & Fuel & & No. of & & BROKEN & \multicolumn{2}{|c|}{ ENCAPSULATION } & \multicolumn{2}{|c|}{ Gas Tran Level lin.I } & LOCKING & \\
\hline Cube & S.N. & Kex & type & modelist & AssYY & Grada & EUEE & Date & YEARS & $M$ & $\underline{u}$ & BARS & \%Pu240 \\
\hline 0603 & $1443 x$ & 13859 & MIAA & $A M$ & 14 & & & Mar-83 & 13.5 & 1.5 & 0.5 & & 11.91 \\
\hline 0661 & $1599 \mathrm{Y}$ & 13859 & MIA & $\overline{A M+A T}$ & $8+6$ & & & Feb-83 & 13.6 & 2.1 & 12.8 & & 11.91 \\
\hline 0719 & $1265 x$ & 12639 & MIA & $A M$ & 14 & & 1 inner & Oct +82 & 13.9 & & & & 12.6 \\
\hline 1264 & 7818 & 15459 & MIA & $\overline{A M+A T}$ & $7+7$ & & & Sep-89 & 7.0 & 16.4 & 1,6 & $B, M$ & 4.01 \\
\hline 2071 & 8139 & 13016 & MIA & $A M$ & 14 & & & Dec-89 & 6.7 & 3.1 & 16.0 & $B, U$ & 12.82 \\
\hline 2233 & $6889 / 99$ & 15244 & MIA & $\overline{A M}+A \bar{T}$ & $2+1$ inner & & chips & Dec-89 & 6.7 & 1.6 & 15.0 & & 10.4 \\
\hline 2241 & 7393 & 15347 & MIA & $A F+A M+A T$ & $1+3+1$ & & clips & Dec-89 & 6.7 & 16.8 & 1.2 & $\overline{C, M}$ & 10.4 \\
\hline 2317 & $696 Y$ & 13686 & MIA & AM & 14 & & & Apr-83 & $\overline{13.4}$ & 3.1 & 0.9 & & 12.03 \\
\hline $2325^{\circ}$ & $738 x$ & 13686 & MIA & AM & 14 & & & $A p r-B 3$ & 13.4 & 3.9 & 13.6 & & 12.03 \\
\hline 2750 & $70 \overline{2 B}$ & 10602 & MIA & $\overline{A M}$ & 14 & & 1 outer & Sep-84 & 12.0 & 9.3 & 1.2 & & 10.9 \\
\hline $3134^{\circ}$ & 8705 & 10456 & MIA & $A M+A T$ & $5+9$ & & 1 inner & OCl-84 & 11.9 & 14.7 & 2.5 & & 11.1 \\
\hline 3355 & 7102 & 10350 & MIA & AM & 14 & & 1 nuter & Oct-84 & 19.9 & 15.4 & 3.4 & & 10.3 \\
\hline 3545 & 7913 & 10350 & $\mathrm{MIA}$ & $\mathrm{AM}$ & 14 & & 2 outers & Oct-84 & 11.9 & 2.4 & 0.9 & & 10.3 \\
\hline 3740 & 6743 & 10982 & MIA & $A M$ & 14 & & & Sep-84 & 12.0 & 3.1 & 16.8 & B.u & 93.24 \\
\hline 4511 & 5744 & 19183 & MIA & $A M$ & 14 & & 1 inner & Jut-84 & 12.2 & 3.4 & 2.2 & & 12.9 \\
\hline 4557 & 6513 & 11109 & $M ! A$ & $\overline{A M}$ & 14 & & 1 outer & Jul-84 & 12.2 & 1.7 & 2.9 & & 13.2 \\
\hline 0856 & 4553 & 13858 & MIV & $B E+B S$ & $8+6$ & & & Nov-83 & 12.8 & 16.3 & 6.7 & $\overline{B E}, M$ & 11.58 \\
\hline 5424 & 1512 & 13858 & MIV & $\mathrm{BE}$ & 14 & & & Mar-83 & 13.5 & 1.9 & 2.2 & & 11.58 \\
\hline 5427 & 91 & 13858 & MIV & $\mathrm{BE}$ & 14 & & & Mar-83 & 13.5 & 3.2 & 2.9 & & 11.58 \\
\hline 5625 & 6214 & 11540 & MIV & $\mathrm{BE}$ & 14 & $\overline{0}$ & & & & 7.4 & 15.8 & & 16.9 \\
\hline 5646 & 5197 & 12852 & MIV & $8 E$ & 14 & 0 & & & & 0.9 & 0.7 & & 15.9 \\
\hline 5652 & 6603 & 11540 & MIV & $B E$ & 14 & D & & & & 1.9 & 16.6 & C.U & 17.0 \\
\hline 5659 & 6641 & 12565 & Miv & BE & 14 & D & & & & 16.2 & 0.8 & $\mathrm{~B} . \mathrm{M}$ & 15.42 \\
\hline 5667 & 6005 & 12565 & MiV & $\mathrm{BE}$ & 14 & D & & & & 1.0 & 0.5 & & 15.6 \\
\hline 6013 & 6082 & 10001 & MIV & $\mathrm{BE}+\mathrm{BA}$ & $1+9$ anter & & chips & Feb-68 & 8.5 & 1.9 & 16.6 & C.U & 9.7 \\
\hline 6014 & 6768 & 10001 & MIV & $B E+B S$ & $6+1$ inner & & chips & Feb-88 & 8.5 & 1.5 & 2.2 & & 9.7 \\
\hline 6016 & 5903 & 10003 & MIV & $\mathrm{AC}+\mathrm{BE}+\mathrm{BS}$ & 1 out $+4+1$ & & chips & Feb-88 & 8.5 & 0.9 & 0.4 & & 9.7 \\
\hline & & & & & & & & & & & & & \\
\hline & & & & & & & & & & & & & \\
\hline & & & & & & & & & & & & & \\
\hline & & & & & & & & & & & & & \\
\hline & & & & & & & & & & & & & \\
\hline
\end{tabular}


HNF-SD-SNF-SM-006, Rev. 0

A P PENDI X B

RECOMMENDED CANISTERS FOR SLUDGE DEPTH MEASUREMENTS AND VISUAL EXAMINATIONS 
DE\&S Hanford, Inc.

A Duke Engineering \& Services Company

Internal

Memo

From: Spent Nuclear Fuel Evaluations

Phone: $\quad 376-5447$ HO-40

Date: October 25,1996

Subject: RECOMMENDED $K$ WEST BASIN CANISTERS FOR ULTRASONIC SLUDGE DEPTH MEASUREMENTS AND FUEL ELEMENT VISUAL EXAMINATIONS

To: R. P. Omberg HO-40

Cc: Distribution

Attached is a list of recommended canisters for the fuel element visual examination campaign in $\mathrm{K}$ West Basin. Canister siudge depth will al so be determined via ultrasonic techniques. The two tables presented in the attachment are for MK I and MK II canisters respectively. Included are 23 prime candidates. Various candidates contain MK IA fue1, MK IV fuel, chips, D Grade fuel, known broken elements, water in-leakage (i.e., broken jocking bars), and/or gas filled traps (suggesting corrosion).

Liquid samples have already been obtained for all of the listed canisters. It is anticipated that a review of visual examination data will be performed after 10 canisters are inspected to ensure that element handling does not prejudice future attempts to obtain sludge samples. Canisters listed in the attached tables are prioritized to facilitate this review and Cs contents (furnished by Dennis Trimble) are included. Archive pictures of several candidate canisters have been obtained by Al Pitner and Wendell Briggs from as-loaded records (circa 1981 through 1983) and are attached for future comparisons to the new visual data.

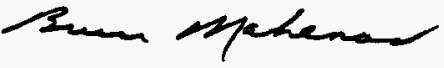

B. J. Makenas

jmn

Attachment

CONCURRENCE:

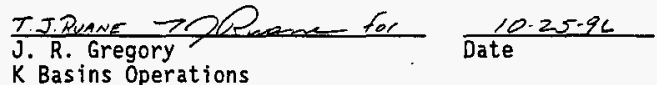

K Basins Operations 
HNF-SD-SNF-SM-006, Rev. 0

ATTACHMENT

Consisting of 7 pages, including cover page 
MK I CANISTERS

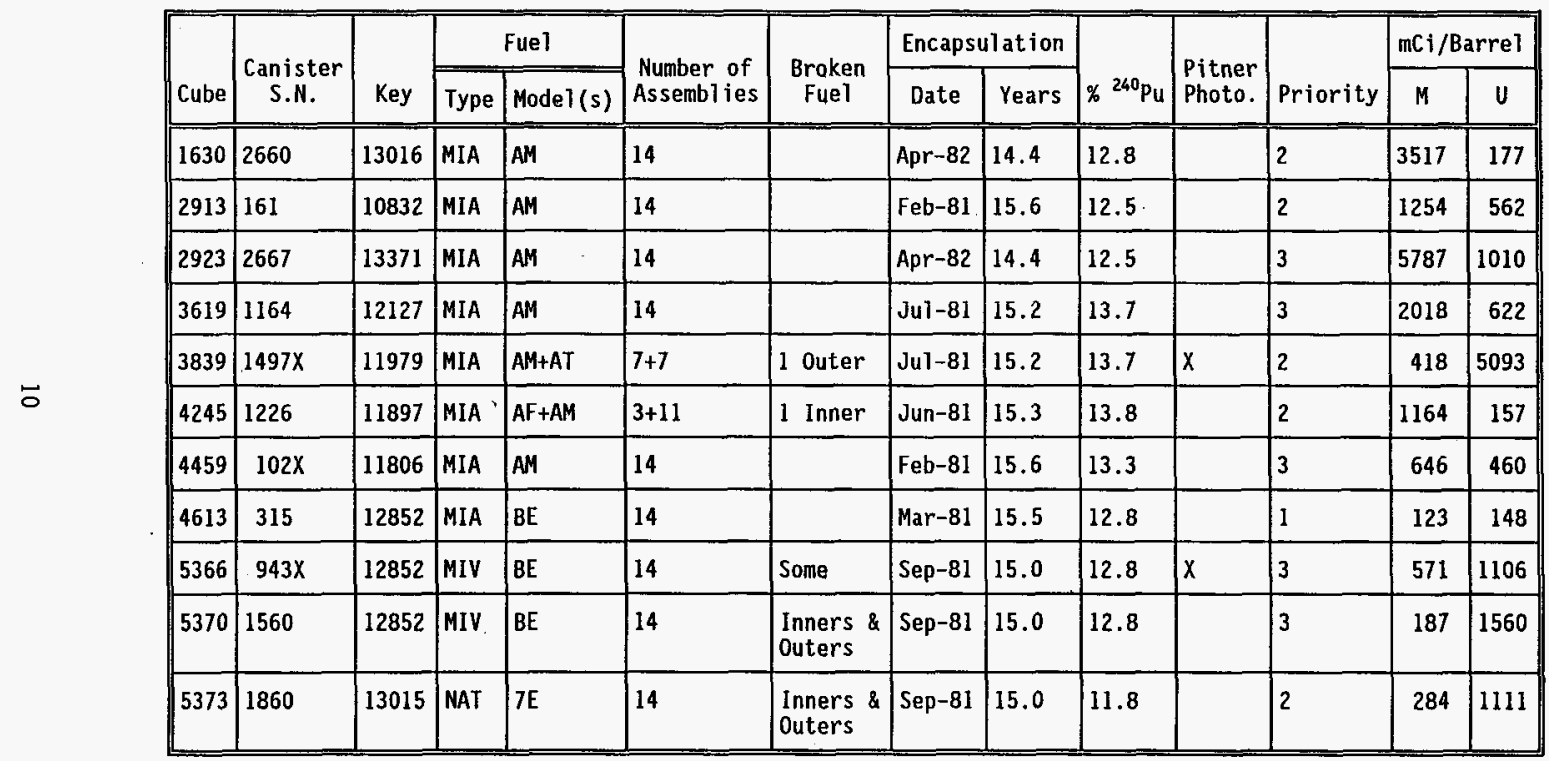


MARK II CANISTERS

\begin{tabular}{|c|c|c|c|c|c|c|c|c|c|c|c|c|c|c|c|c|c|}
\hline & & & & Fuel & & & & Encapsu & ulation & $\begin{array}{r}\text { Gas } \\
\text { Le } \\
\text { (i }\end{array}$ & $\begin{array}{l}\text { Trap } \\
\text { vel } \\
\text { n.) }\end{array}$ & & & & & $\underset{\text { Bar }}{m C}$ & $\begin{array}{l}1 / \\
\text { rel }\end{array}$ \\
\hline Cube & S.N. & Key & Type & Hodel (s) & Assemblies & Grade & Fuel & Date & Years & M & u & Bars & $\approx{ }^{240} \mathrm{Pu}$ & Photo. & Priority & H & u \\
\hline 0719 & $1265 x$ & 12639 & MIA & AM & 14 & & 1 Inner & oct-82 & 13.9 & & & & 12.6 & & 3 & 465 & 728 \\
\hline 2233 & 6889 & 15244 & MIA & AN+AT & $2+1$ Inner & & Chips & Dec-89 & 6.7 & 1.6 & 15.0 & & 10.4 & & 2 & 870 & 45 \\
\hline 3545 & 7913 & 10350 & NIA & AM & 14 & & 2 Duter & oct -84 & 11.9 & 2.4 & 0.9 & & 10.3 & & 3 & 358 & 1127 \\
\hline 3740 & 6743 & 10982 & MIA & AM & 14 & & & Sep-84 & 12.0 & 3.1 & 16.8 & $B, U$ & 13.24 & $x$ & 2 & 935 & $?$ \\
\hline 4511 & 5744 & 11183 & MIA & AM & 14 & & 1 Inner & Jul-84 & 12.2 & 3,4 & 2.2 & & 12.9 & & 2 & 840 & 1320 \\
\hline 4557 & 6513 & 11109 & MIA & AM & 14 & & 1 Outer & Jui-84 & 12.2 & 1.7 & 2.9 & & 13.2 & $x$ & 3 & 4419 & 1671 \\
\hline 5424 & 1512 & 13858 & MIV & BE & 14 & & & Mar-83 & 13.5 & 1.9 & 2.2 & & 11.58 & & 3 & 932 & 221 \\
\hline 5427 & 91 & 13858 & HIV & BE & 14 & & & Mar-83 & 13.5 & 3.2 & 2.9 & & 11.58 & & 3 & 770 & 661 \\
\hline 5625 & 6214 & 11540 & MIV & $B E$ & 14 & 0 & & & & 7.4 & 15.8 & & 16.9 & & 1 & 122 & 125 \\
\hline 5652 & 6603 & 11540 & HIV & $B E$ & 14 & 0 & & & & 1.9 & 16.6 & $c, v$ & 17.0 & & 3 & 766 & 204 \\
\hline 6014 & 6768 & 10001 & MIV & $B E+85$ & $6+1$ Inner & & Chips & Feb-88 & 8.5 & 1.5 & 2.2 & & 9.7 & & 3 & 2582 & $?$ \\
\hline 6016 & 5903 & 10001 & MIV & $B C+B E+B S$ & 1 out $+4+1$ Out & & Chips & Feb-88 & 8.5 & 0.9 & 0.4 & & 9.7 & & 3 & 4688 & 4141 \\
\hline
\end{tabular}

S.N. - Serial Number, C = Cracked, B = Broken, BE = Broken End, M = Marked Barrel, U = Unmarked Barrel, 1 - Perform First 
HNF-SD-SNF-SM-006, Rev. 0

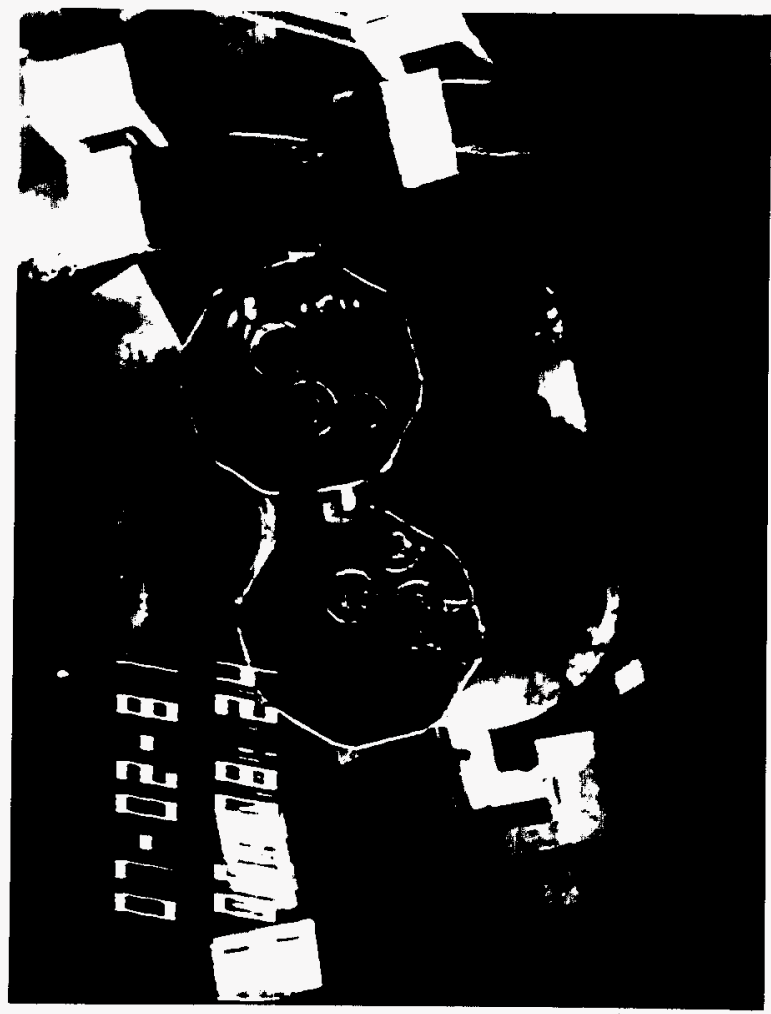


HNF-SD-SNF-SM-006, Rev. 0

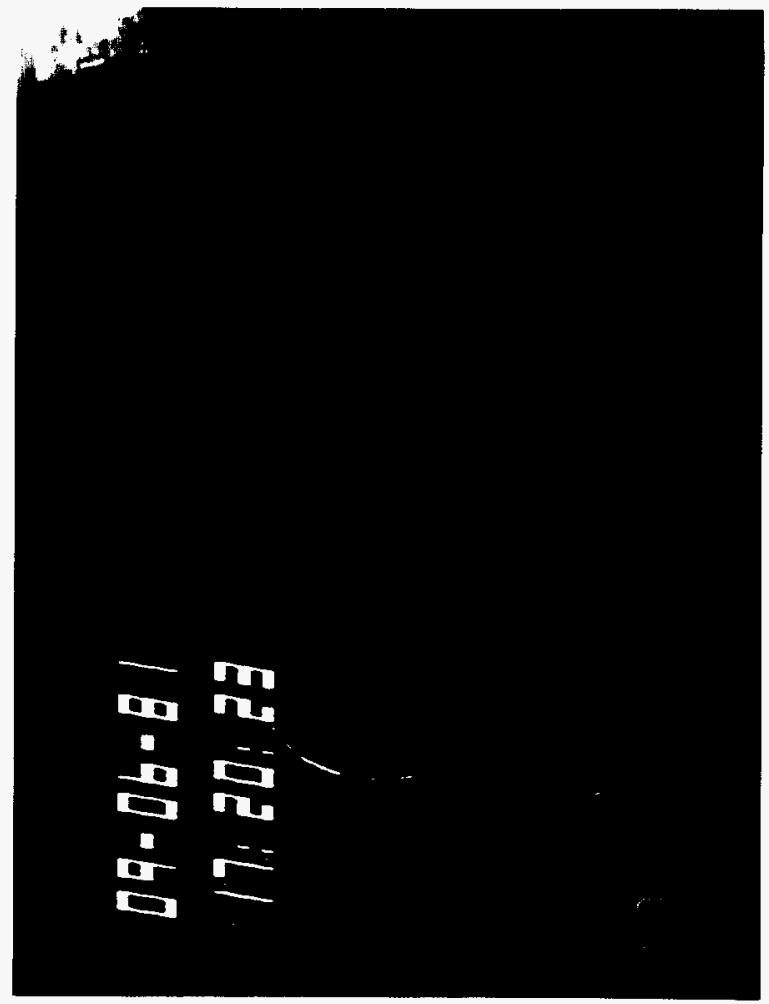


HNF-SD-SNF-SM-006, Rev. 0

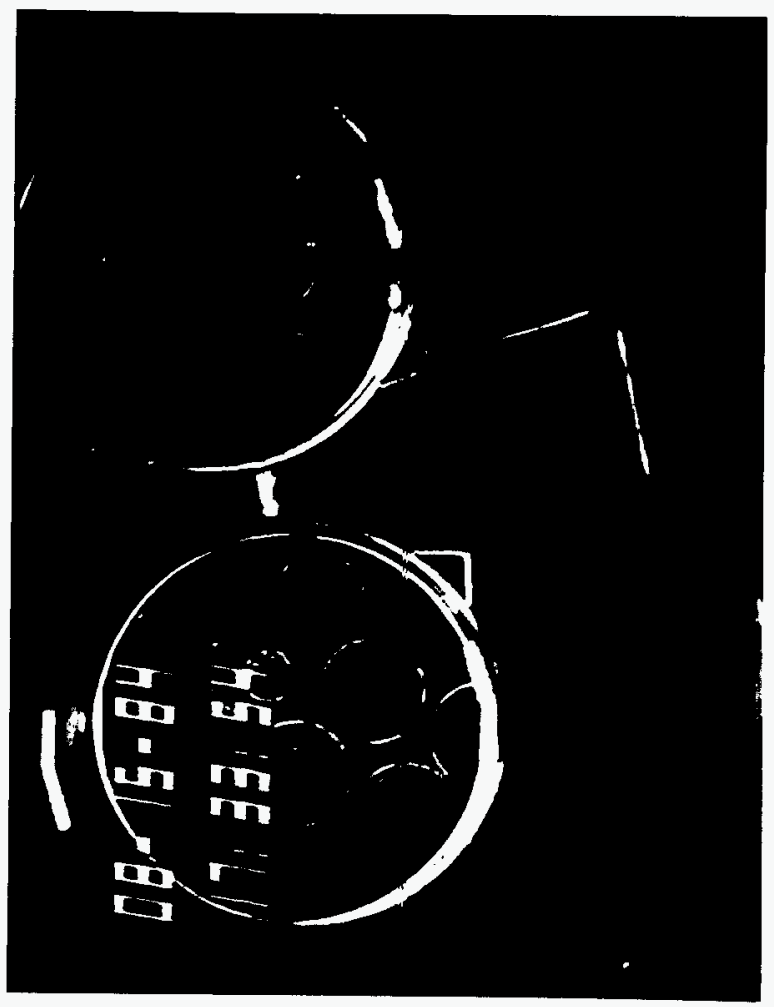


HNF-SD-SNF-SM-006, Rev. 0

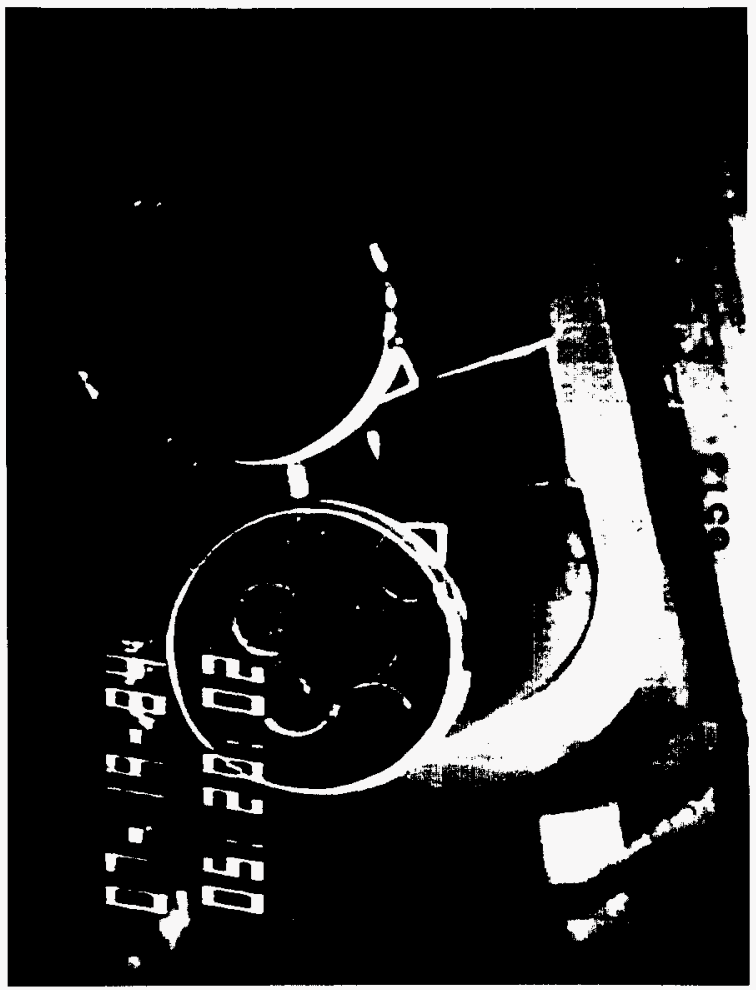


HNF-SD-SNF-SM-006, Rev. 0

This page intentionally left blank. 
HNF-SD-SNF-SM-006, Rev. 0

A P PENDIX C

RECOMMENDED CANISTERS FOR SLUDGE SAMPLING 
DE\&S Hanford, Inc.

A Duke Engineering \& Services Company

Internal

Memo

From: $\quad$ Spent Nuclear Fuel Evaluations

Phone: $376-5109$ H0-40

Date: December 6, 1996

Subject: RECOMMENDED $\mathrm{K}$ WEST BASIN CANISTERS FOR SLUDGE SAMPLING

To: $\quad$ R. P. Omberg HO-40

cc: Distribution

Attachment I provides a list of the nine recommended canister barrels for sludge sampling in the $K$ West Basin. This recommendation was developed considering results from (1) the recent analyses of samples of liquid from selected sealed canisters, (2) the sludge depth data determined by ultrasonic techniques, and (3) the recent visual examination of fuel elements. D. J. Trimble provided major support in integrating the results of the liquid sampling results and the general parameters involved. A. L. Pitner and W. A. Briggs provided initial evaluation of the visual examination video tape data (i.e., 72 tapes covering examination of 48 canjster barrels of spent fuel).

The data were reviewed by A. L. Pitner, D. J. Trimble, B. J. Makenas, and R. B. Baker to develop this recommendation consistent with objectives of $K$ West sludge characterization. The parameters considered included cesium-137 activity in the liquid taken from the sealed canisters, calculated sludge volume in the barrels based on ultrasonic measurements of depth, the observed evidence of sludge volume based on visual examinations of fuel elements, canister type (e.g., Mark I, Mark II, aluminum, stainless steel, etc.), fuel condition (e.g., age, burnup, number of breaches, and reactions), and fuel type (e.g., Mark IA, Mark IV, D Grade, etc.).

Attachment 2 summarizes some of the primary parameters.

As a contingent, Attachment 1 also includes a selected backup canister barrel that should be sampled if major problems are encountered with the nine recommended samples.

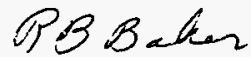

R. B. Baker

jmn

Attachment

CONCURRENCE:

BY $M$

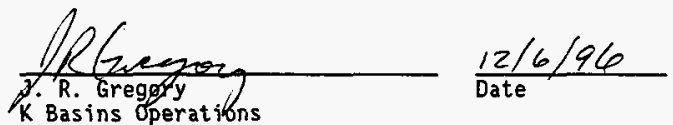

Reference:B.J.Makenas, K.L.Pearce and R.B.Baker, "Data Quality Objectives for K West Basin Canister Sludge Sampling", WHC-SD-SNF-DQ0-012, Rev. 0, December 1996. 
HNF-SD-SNF-SM-006, Rev. 0

ATTACHMENT 1

RECOMMENDED $K$ WEST BASIN CANISTER BARRELS FOR SLUDGE SAMPLING

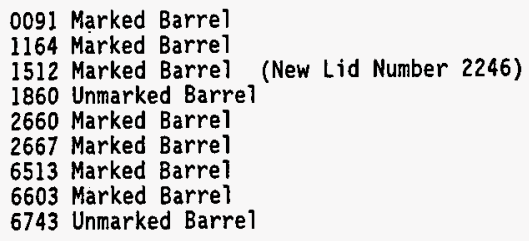

Backup Canister Choice: 5744 Marked Barrel 
HNF-SD-SNF-SM-006, Rev. 0

ATTACHMENT 2

SELECTED PARAMETERS FOR CANISTER BARRELS RECOMMENDED FOR SLUDGE SAMPLING

\begin{tabular}{|c|c|c|c|c|c|c|c|c|c|c|c|c|}
\hline \multirow{2}{*}{\multicolumn{3}{|c|}{\begin{tabular}{|c|c|c|}
\multicolumn{3}{|c|}{ CANISTER } \\
Darrel
\end{tabular}}} & \multirow{3}{*}{$\begin{array}{c}\text { Cs137 } \\
\text { Cimarted }\end{array}$} & \multicolumn{3}{|c|}{ SLUDGE } & \multicolumn{4}{|c|}{ FUEL } & \multirow{3}{*}{$\begin{array}{c}\text { before } \\
\text { encapsulaled } \\
\text { (vis) }\end{array}$} & \multirow{3}{*}{$\begin{array}{c}\text { affer } \\
\text { encspsulated } \\
\text { (vas) }\end{array}$} \\
\hline \multicolumn{3}{|c|}{ CANISTER } & & \multirow{2}{*}{$\begin{array}{l}\text { depth } \\
\text { finchi }\end{array}$} & \multirow{2}{*}{ volume } & \multirow{2}{*}{$\begin{array}{l}\text { qualitative } \\
\text { observallon }\end{array}$} & \multicolumn{3}{|c|}{ 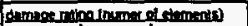 } & \multirow[b]{2}{*}{ type } & & \\
\hline & & malerial & & & & & miner & maior & yery miolor & & & \\
\hline $0001 M$ & MKII & S.S. & 0.77 & 2.5 & 0.78 & 0000 & 7 & $\overline{1}$ & $T$ & MKIA & 0.5 & 13.5 \\
\hline $1964 M$ & MKI & s.s. & 2.02 & 3.1 & 1.01 & moderate & $\frac{1}{1}$ & 1 & 2 & $\overline{M K I A}$ & 3.6 & $1 \overline{5.2}$ \\
\hline $1512 M$ & MKII & s.5. & 0.93 & 0.7 & 0.22 & good & $\overline{3}$ & $\overline{0}$ & 9 & MKIV & 0.5 & 13.5 \\
\hline $1860 \mathrm{U}$ & MKI & Alum. & 1.19 & 0.8 & 0.21 & 0000 & 2 & 2 & 1 & MKIV & 1.3 & 15.0 \\
\hline $2660 \mathrm{M}$ & MKI & S.5. & 3.5 & 1.3 & 0.41 & good & $\overline{0}$ & 9 & 1 & MK IA & 1.9 & 14.3 \\
\hline $2667 \mathrm{M}$ & MKI & S.S. & 5.79 & 0.6 & 0.21 & 0000 & 1 & $\overline{0}$ & 1 & MK'IA & 0.9 & 94.4 \\
\hline $6513 M$ & MK II & S.S. & 4.42 & 0.8 & 0.27 & 9000 & 2 & 0 & 2 & $\overline{M K I A}$ & 9.4 & 12.2 \\
\hline $6603 \mathrm{M}$ & MK II & S.S. & 0.77 & 1.8 & 0.61 & good & 4 & $\overline{1}$ & $\frac{1}{1}$ & MK IV & \multicolumn{2}{|c|}{ unknown (D grede) } \\
\hline $6743 \mathrm{U}$ & MK II & s.S. & no date & 1.4 & 0.46 & 9000 & 9 & D & 2 & MKIA & 9.9 & 12.0 \\
\hline
\end{tabular}


HNF-SD-SNF-SM-006, Rev. 0

\author{
A P PENDIX D
}

RECOMMENDED CANISTERS TO SUPPLY ELEMENTS FOR HOT CELL EXAMINATIONS 
DE\&S Hanford, Inc.

From: $\quad$ Spent Nuclear Fuel Evaluations

Phone: $\quad 376-5447 \quad \mathrm{HO}-40$

Date: January 15,1997

Subject: RECOMMENDED $K$ WEST BASIN CANISTERS FOR FUEL ELEMENT RETRIEVAL (SECOND CAMPAIGN)

To: $\quad$ R. P. Omberg HO-40

cc: Distribution

Reference: L. A. Lawrence, B. J. Makenas, and D. W. Bergmann, "Data Quality Objectives for the Second K West Fuel Examinations, "HNF-SD-SNF-DQO-011, Rev. O, dated January 1997.

The 15 elements 7 isted in Table 1 are recommended to be shipped from $K$ West Basin to 327 Building hot cells for examination. These elements will satisfy the requirements specified in the reference. Elements representing categories such as top and/or bottom failures, severe damage, mid-element cracks, complete break, and unusual coatings have been included. These will feed whole-element drying, thermogravimetric analyses, coating scraping, and visual examinations.

Also specified in Table 1 are number of backup choices in case difficulties are encountered during canister or element handing. Top view pictures of each candidate canister (as seen during the recent visual examination campaign) are attached to aid in element identification.

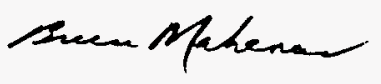

B. J. Makenas

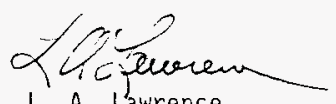

L. A. Lawrence

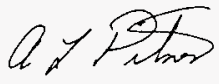

A. L. Pitner

jmn

Attachment

CONCURRENCE:
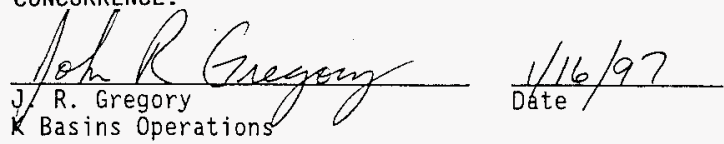
Table 1. Fuel Elements to be Shipped to Hot Cells for the Second $K$ West Basin Fuel Retrieval Campaign.

\begin{tabular}{|c|c|c|c|c|c|c|}
\hline $\begin{array}{c}\text { Canister } \\
\text { Number }\end{array}$ & Location & $\begin{array}{c}\text { Element } \\
\text { Number }\end{array}$ & $\begin{array}{c}\text { Canister } \\
\text { Type }\end{array}$ & Requirement ${ }^{2}$ & $\begin{array}{l}\text { Fue } 1 \\
\text { Type }\end{array}$ & $\begin{array}{c}\text { Fuel } \\
\text { Mode1 } \\
\text { Code } \\
\end{array}$ \\
\hline \multicolumn{7}{|c|}{ Prime Candidates } \\
\hline $161 M^{3}$ & 2913 & 2 & MK I & Coating & MK IA & AM \\
\hline $2667 U^{3}$ & 2923 & 3 & MK I & Coating & MK IA & AM \\
\hline $5744 \mathrm{U}$ & 4511 & 4 & MK II & Broken top end & MK IA & AM \\
\hline $6743 \mathrm{M}$ & 3740 & 6 & MK II & Broken top end & MK IA & AM \\
\hline $6603 \mathrm{M}$ & 5652 & 5 & MK II & Broken bottom end & MK IV & BE \\
\hline $7913 M$ & 3545 & 6 & MK II & Broken bottom end & MK IA & AM \\
\hline $6743 \mathrm{U}$ & 3740 & 6 & MK II & Cladding peal & MK IA & AM \\
\hline $7913 U$ & 3545 & 4 & MK II & Cladding peal & MK IA & AM \\
\hline $1164 M$ & 3619 & 5 & MK I & Mid-element cracks & MK IA & AM \\
\hline $6743 \mathrm{U}$ & 3740 & 2 & MK II & Mid-element cracks & MK IA & AM \\
\hline 11640 & 3619 & 1 & MK I & Mid-element cracks & MK IA & AM \\
\hline $2660 \mathrm{M}$ & $1630 ! 730$ & 6 & MK I & Severely damaged & MK IA & AM \\
\hline $6513 U$ & 4557 & 6 & MK II & Severely damaged & MK IA & AM \\
\hline $6513 \mathrm{M}$ & 4557 & 1 & MK II & Severely damaged & MK IA & AM \\
\hline $0309 \mathrm{M}^{4}$ & 5370 & 6 & MK 1 & Broken in half & MK IV & BE \\
\hline \multicolumn{7}{|c|}{ Backup Candidates } \\
\hline $1497 M^{3}$ & 3839 & 4 & MK I & $\begin{array}{l}\text { Backup coating with top } \\
\text { rupture }\end{array}$ & MK IA & $\begin{array}{l}\text { AM or } \\
\text { AT }\end{array}$ \\
\hline $5744 M^{3}$ & 4511 & 6 & MK II & $\begin{array}{l}\text { Backup coating with top } \\
\text { and bottom rupture }\end{array}$ & MK IA & AM \\
\hline $7913 U$ & 3545 & 5 & MK II & Backup broken bottom & MK IA & AM \\
\hline $1164 M$ & 3619 & 2 & MK I & $\begin{array}{l}\text { Backup top and bottom } \\
\text { rupture }\end{array}$ & MK IA & AM \\
\hline $03090^{4}$ & 5370 & 6 & MK I & Backup clean break & MK IV & $B E$ \\
\hline
\end{tabular}

${ }^{1} \mathrm{CW}$ from trunion with element closest to trunion designated as Number 1.

${ }^{2}$ Backup elements with coincident top and bottom ruptures may be substituted for cladding peal, top or bottom rupture, mid-element cracking, or severely damaged categories.

${ }^{3}$ Canisters containing primary elements for brushing.

${ }^{4}$ Formerly canister 1560 prior to 7 id replacement. 
HNF-SD-SNF-SM-006, Rev. 0

\author{
ATTACHMENT \\ List and Photographs of Candidate Canisters \\ Consisting of 22 pages, \\ including cover page
}




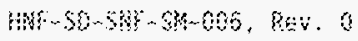

$110 \%$ \& ... W. .
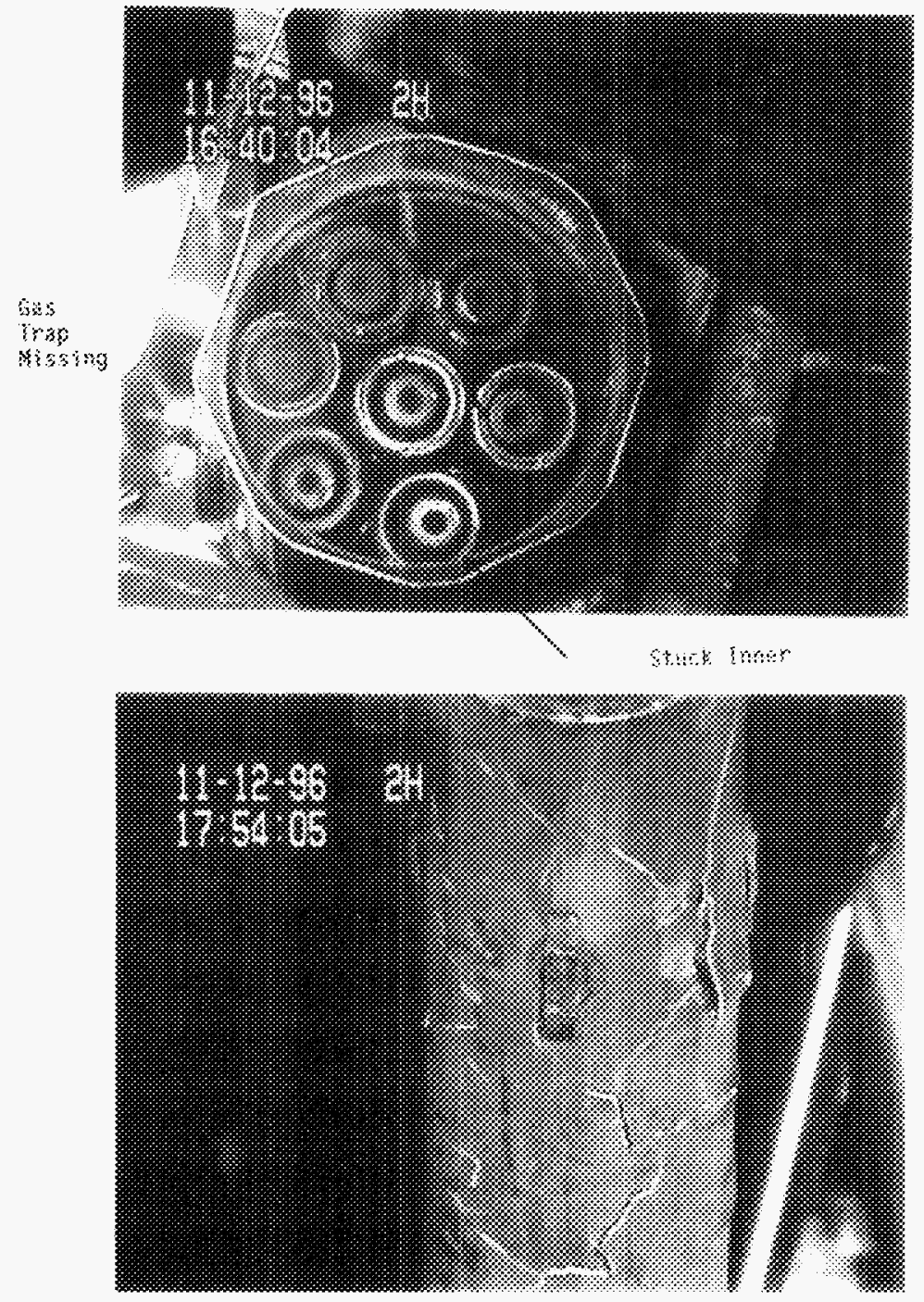


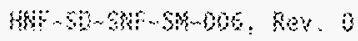

$$
6434 \ldots 3 \% 2
$$
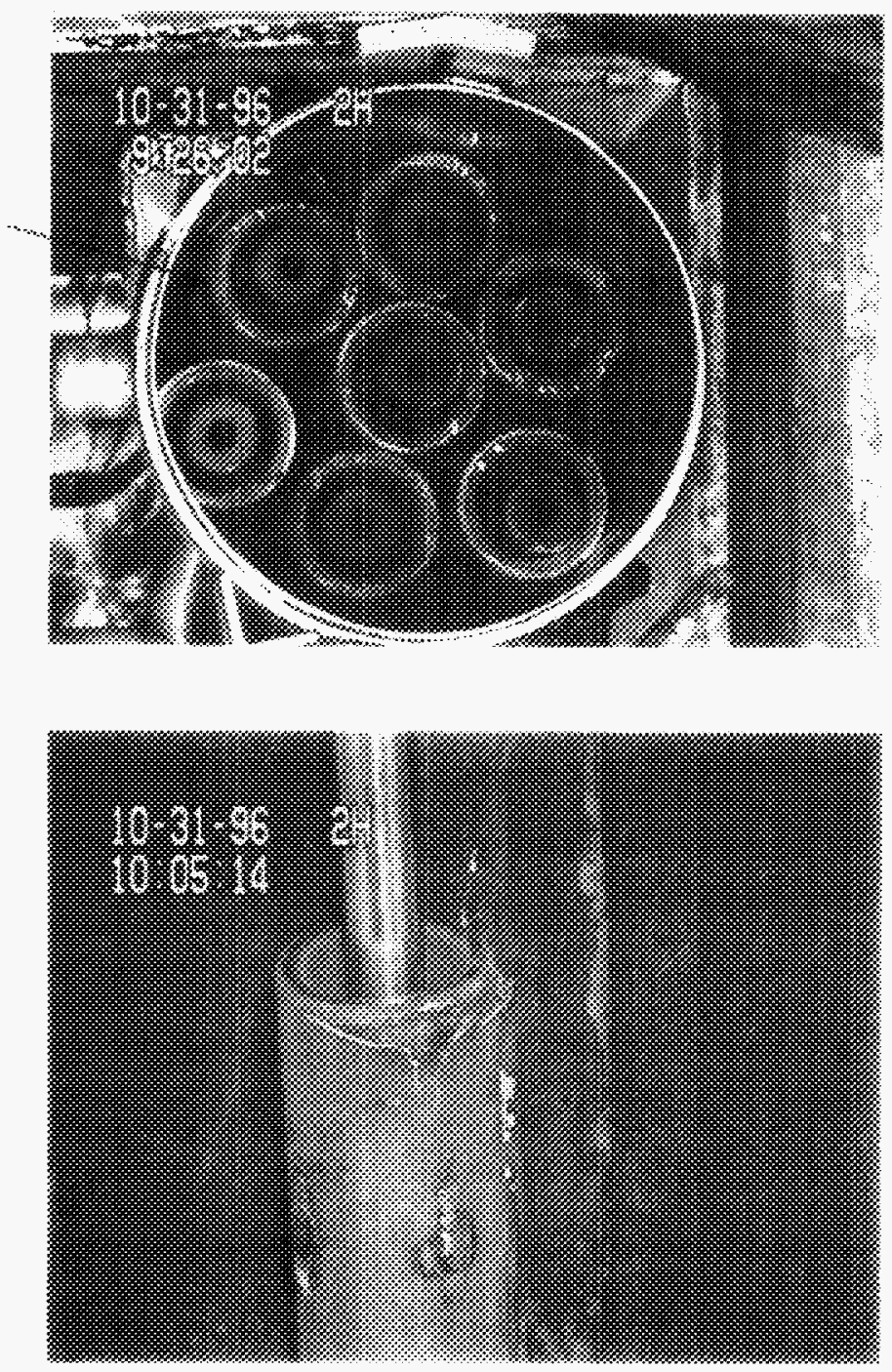


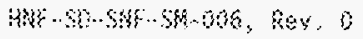

134 ... Wh. 2
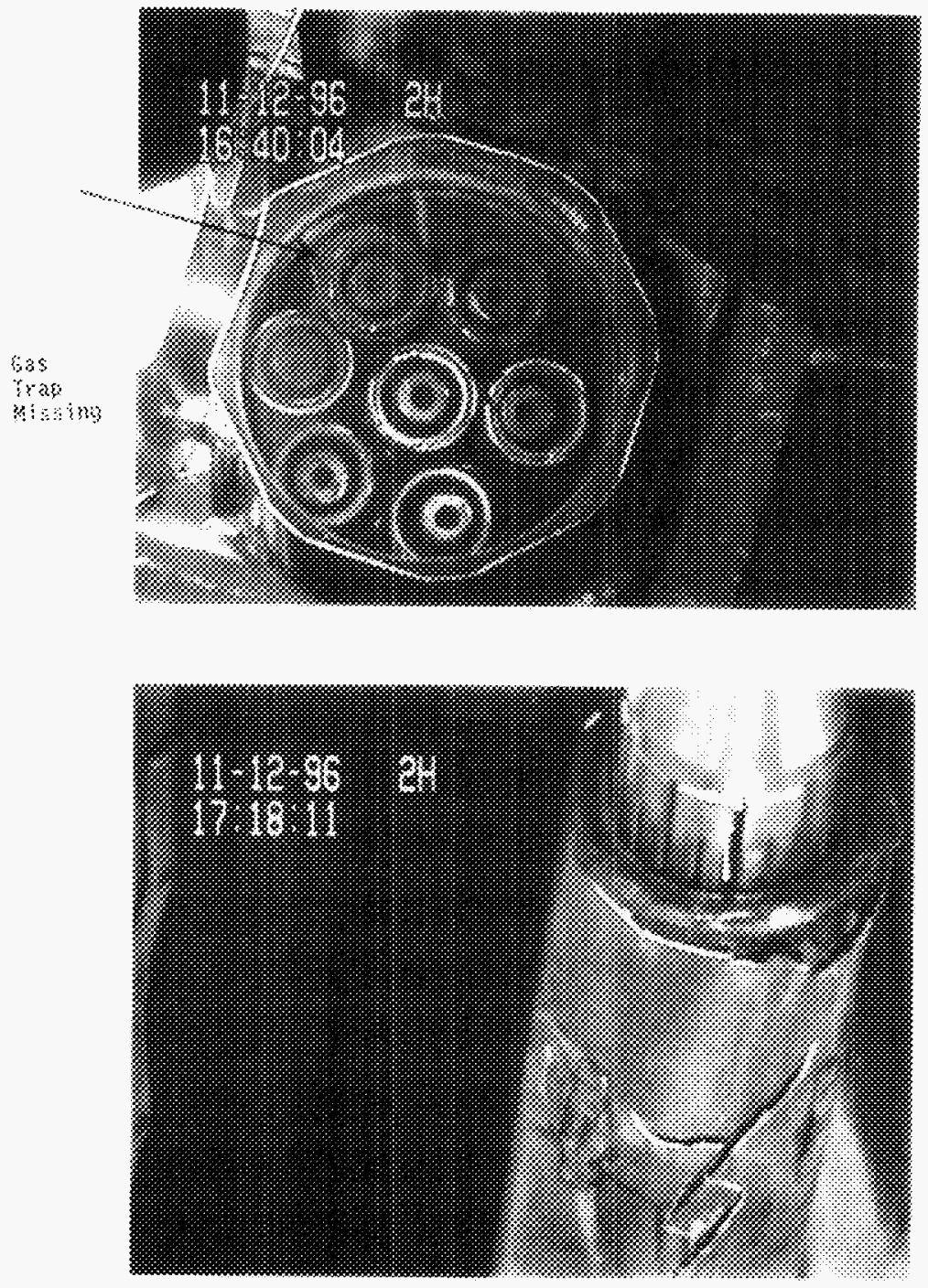


\section{$2680 \mathrm{M} \ldots \mathrm{M} .6$}
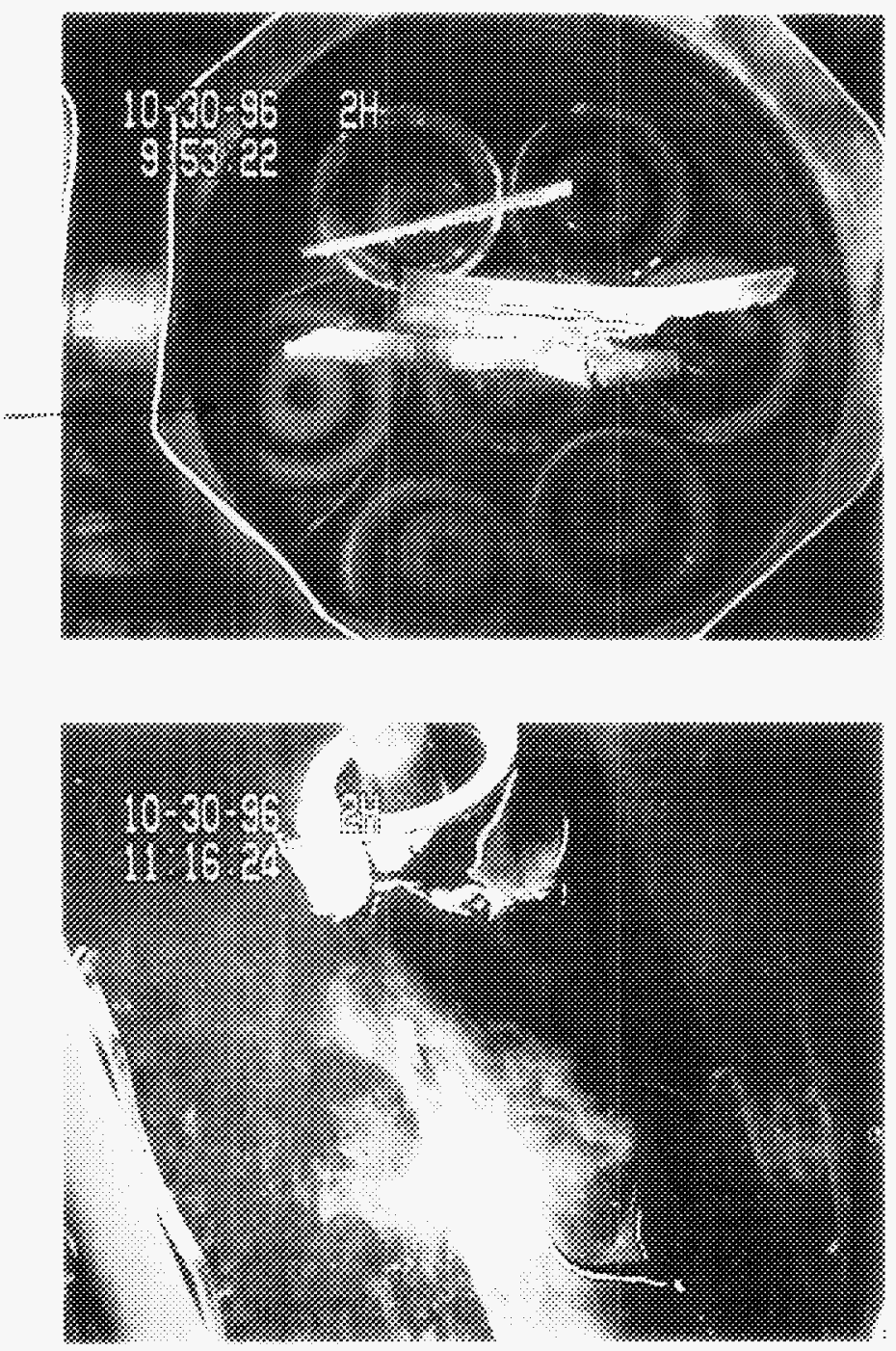

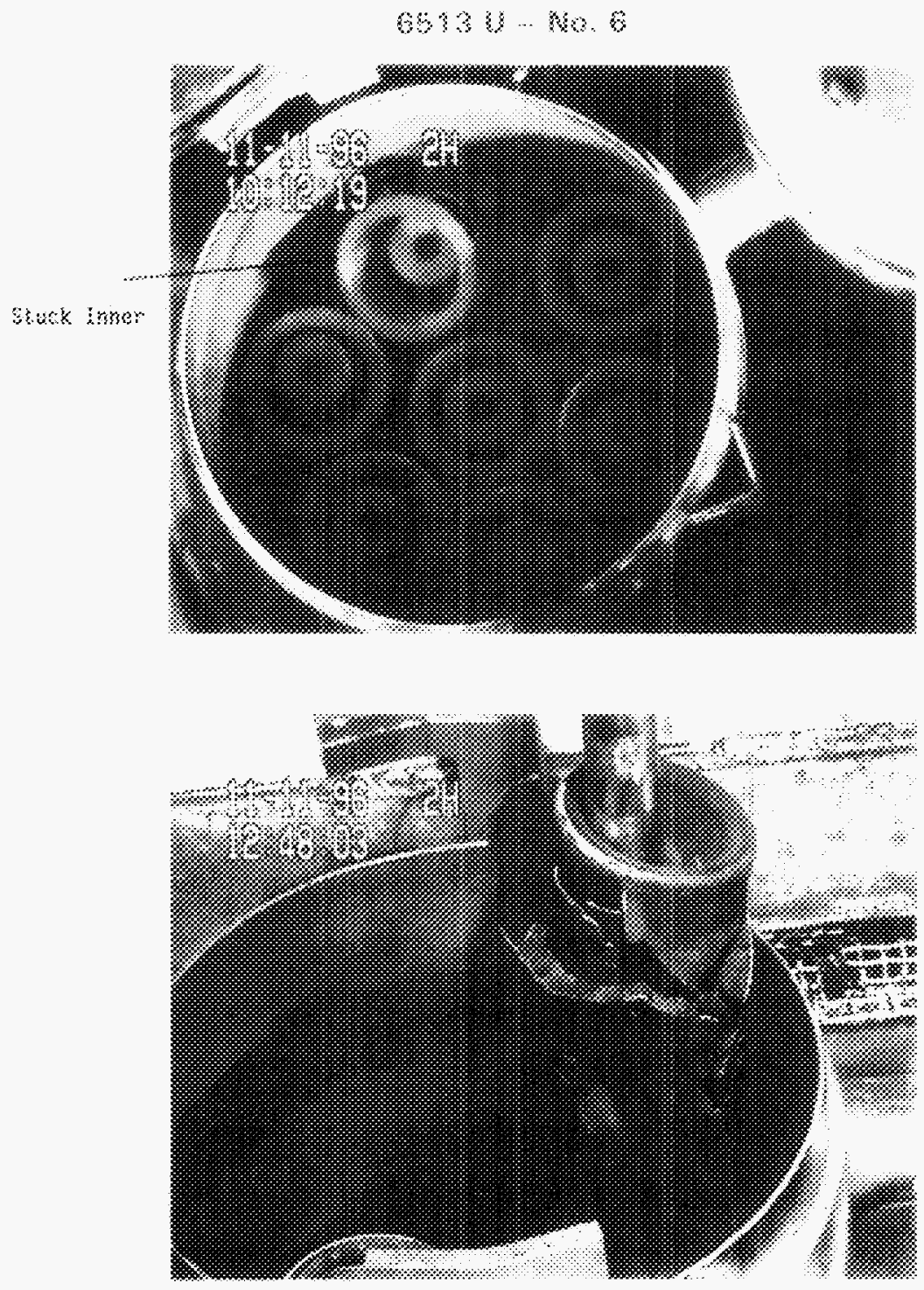
5\%? $\mathrm{KH} \mathrm{Mo}$
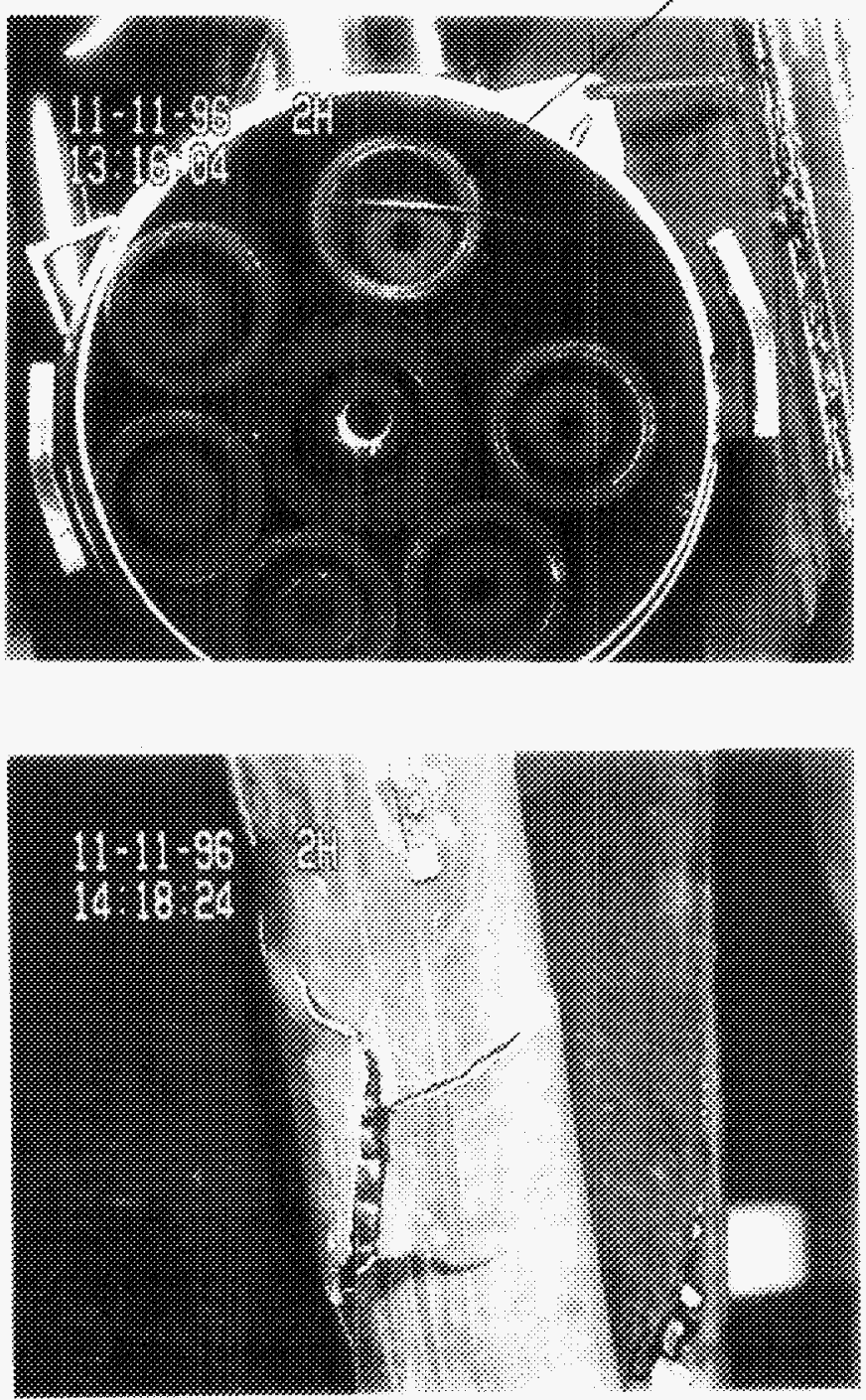

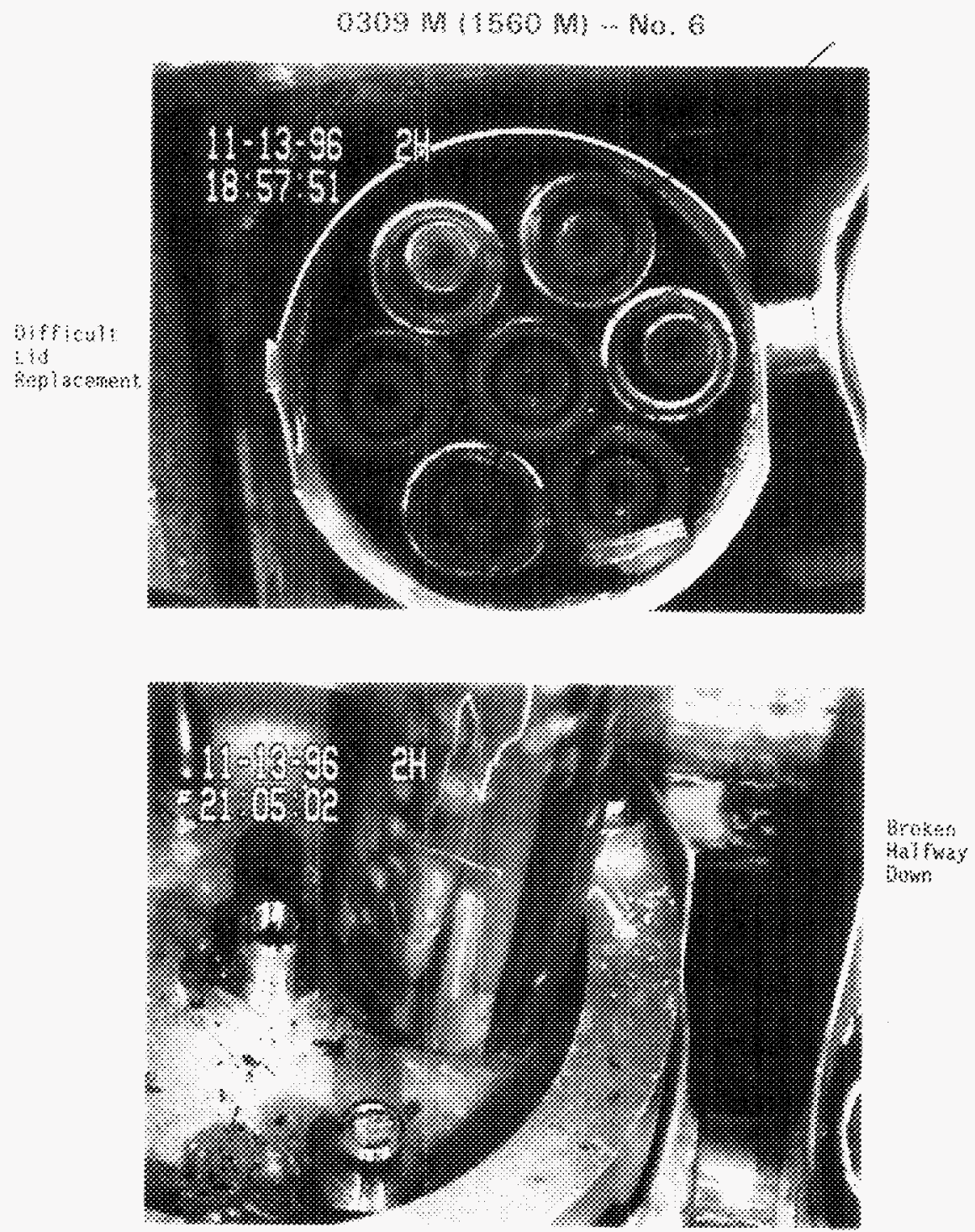


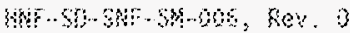

\section{$4093 \%$...}
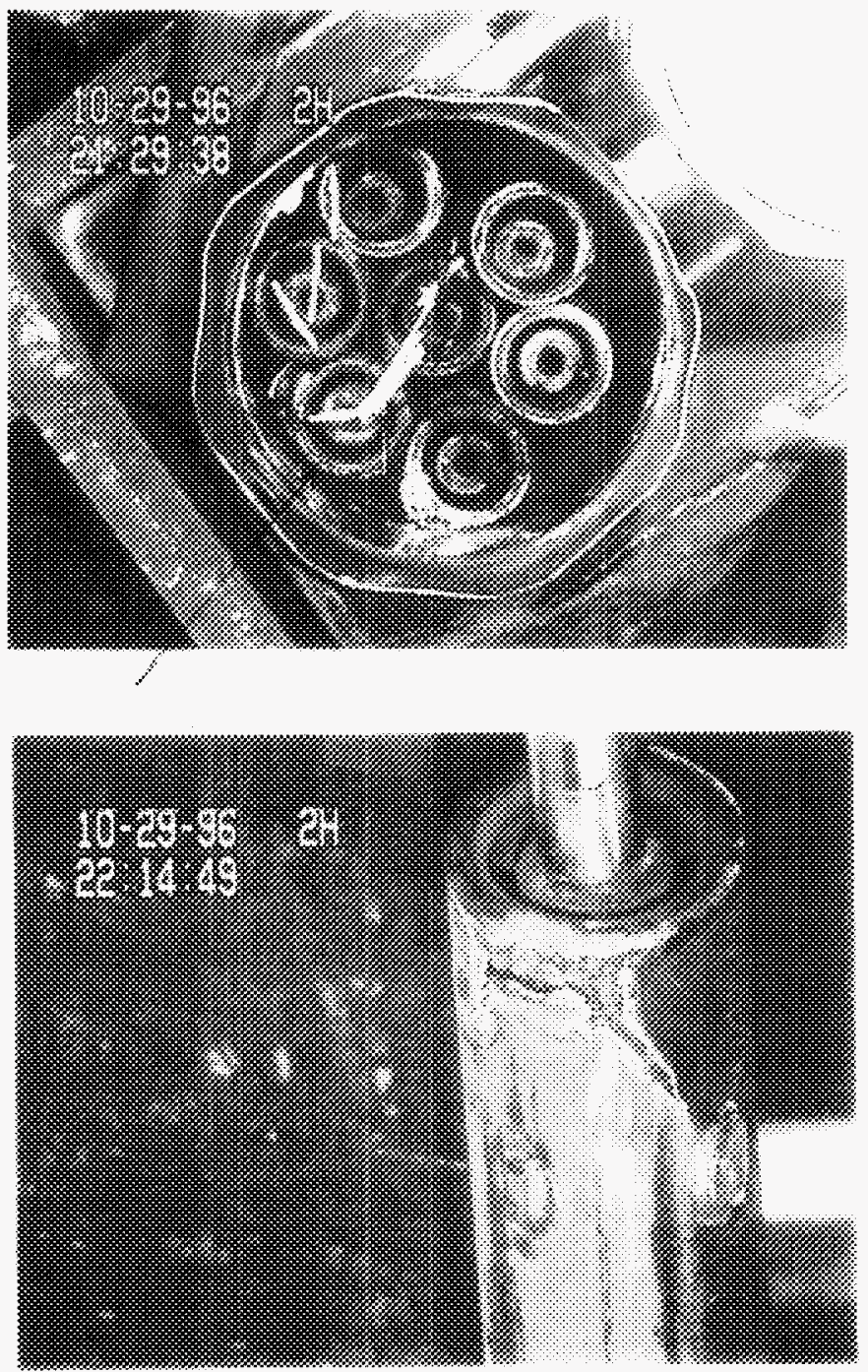
$5 \% 3 \times$. B. \& 8
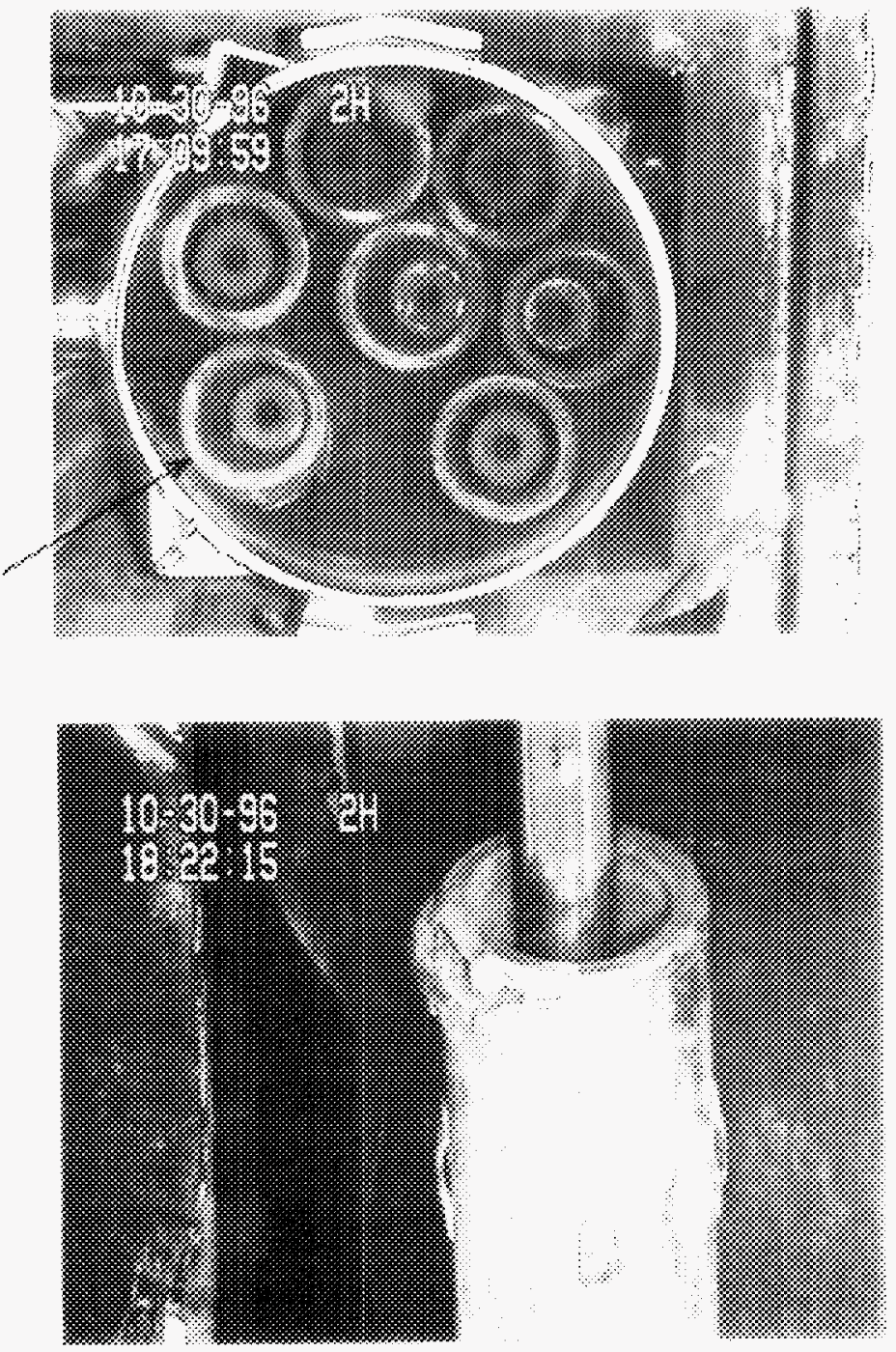


\section{$7913 \div \ldots$}
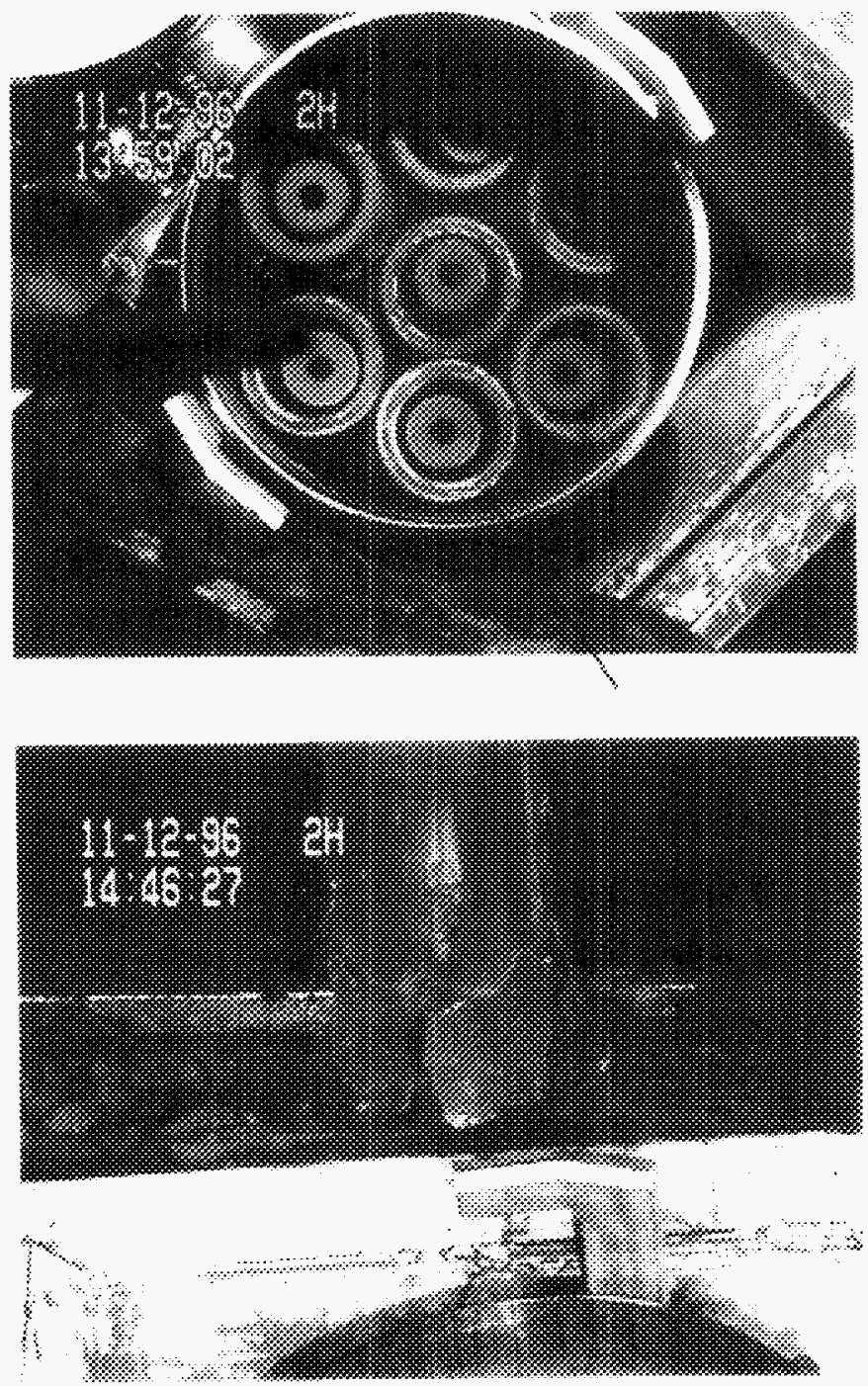

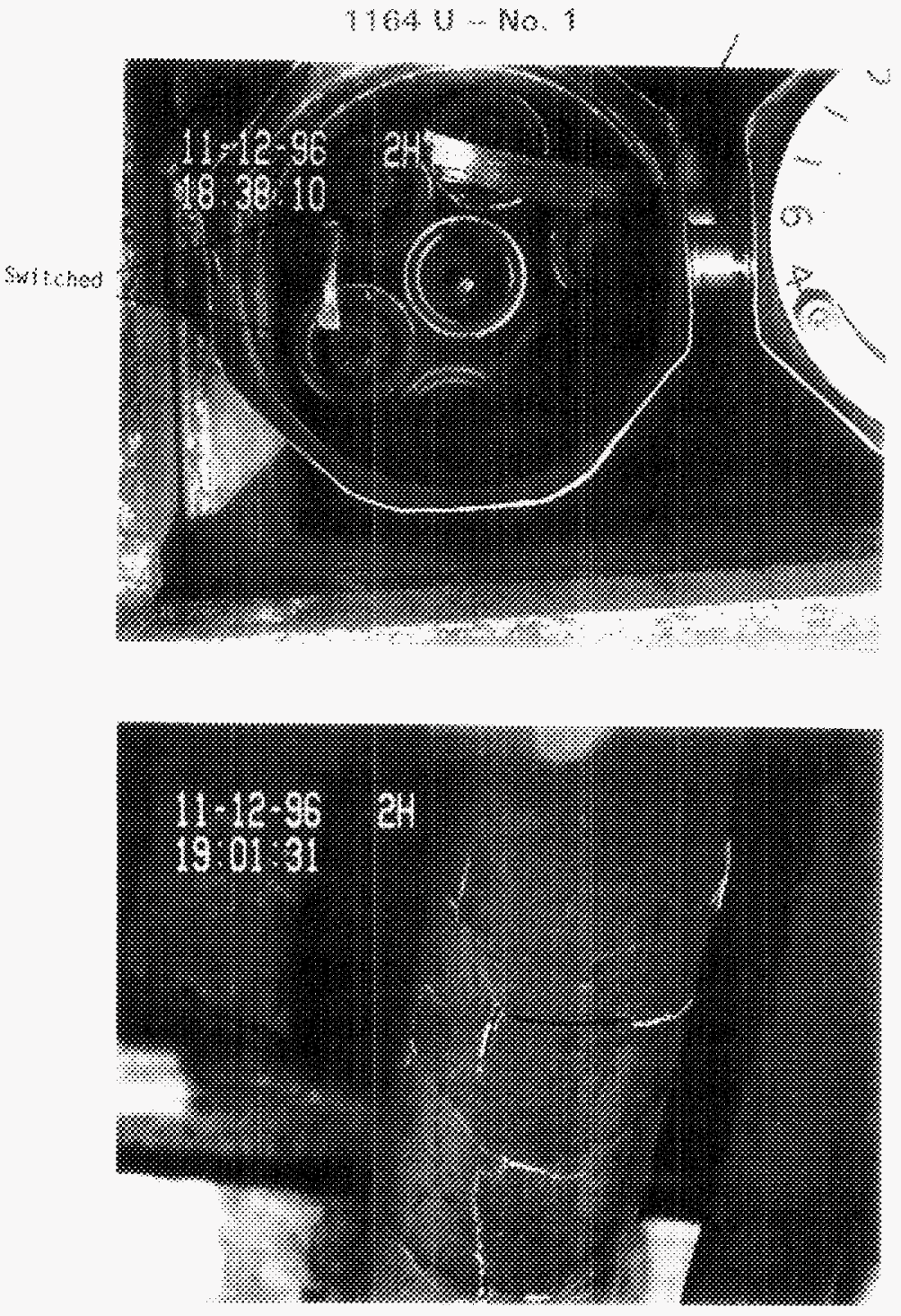


\section{$0309\} ; 3603 \%$.}
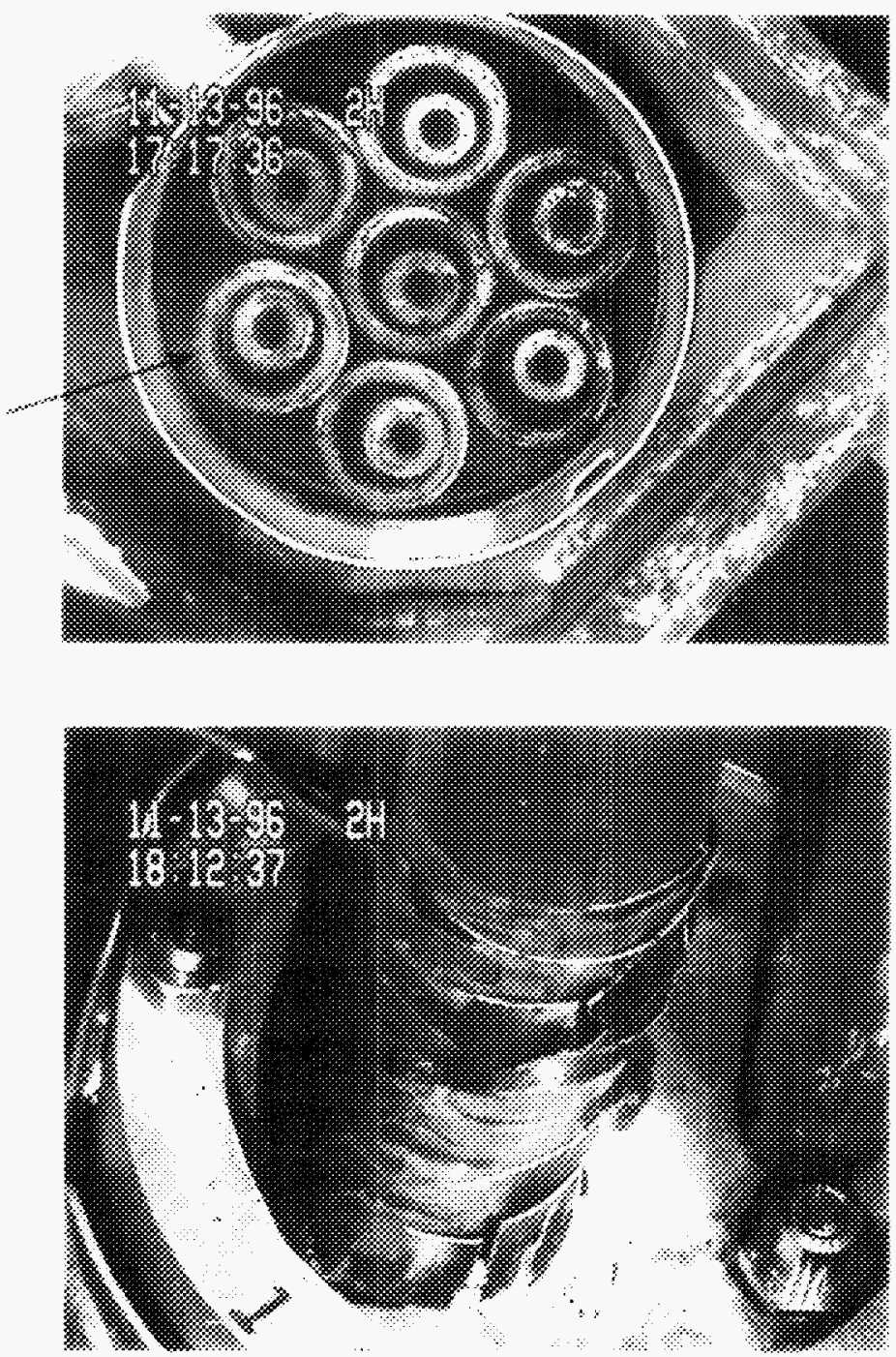


\section{$0314 \ldots .2$}
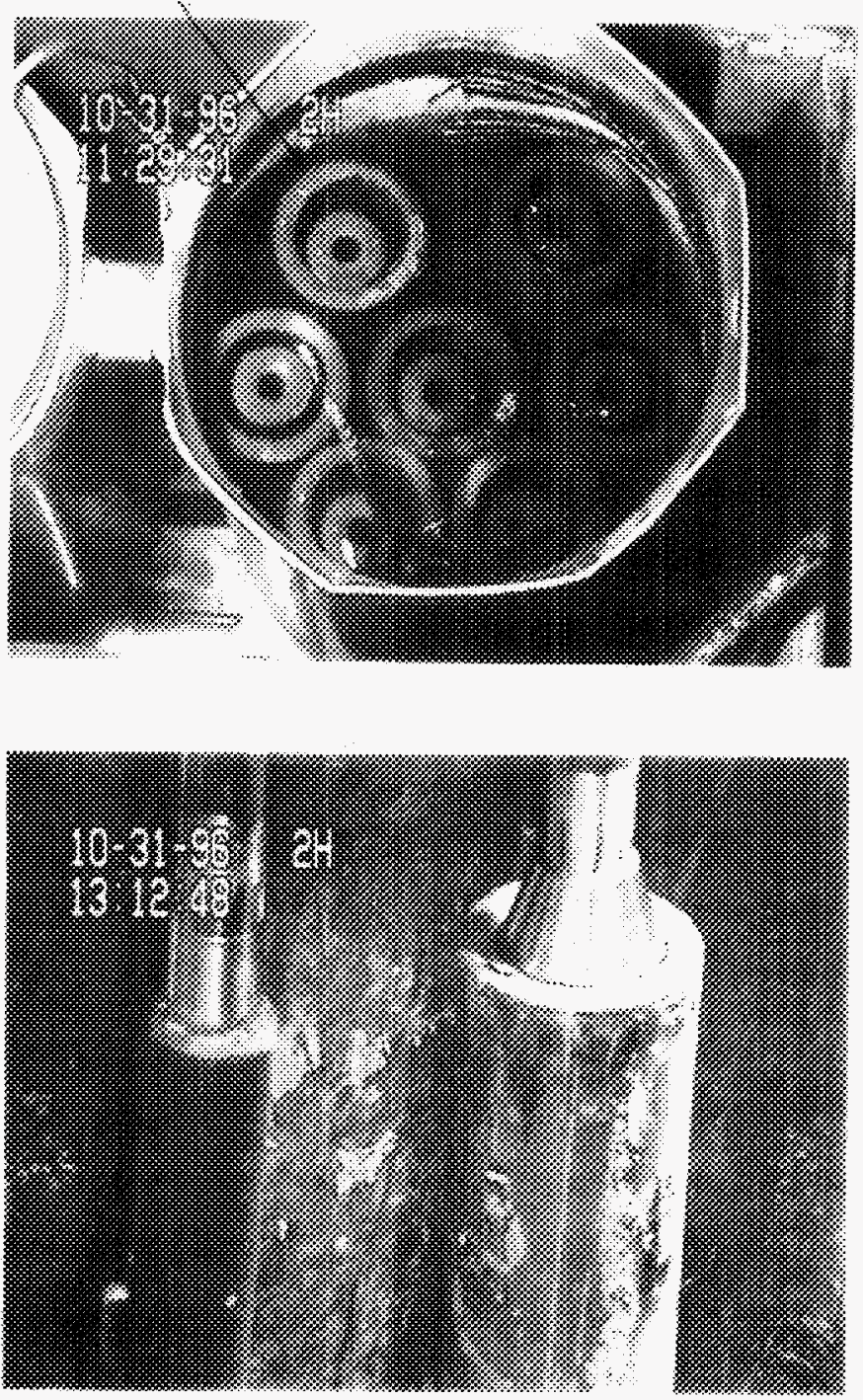
:61-
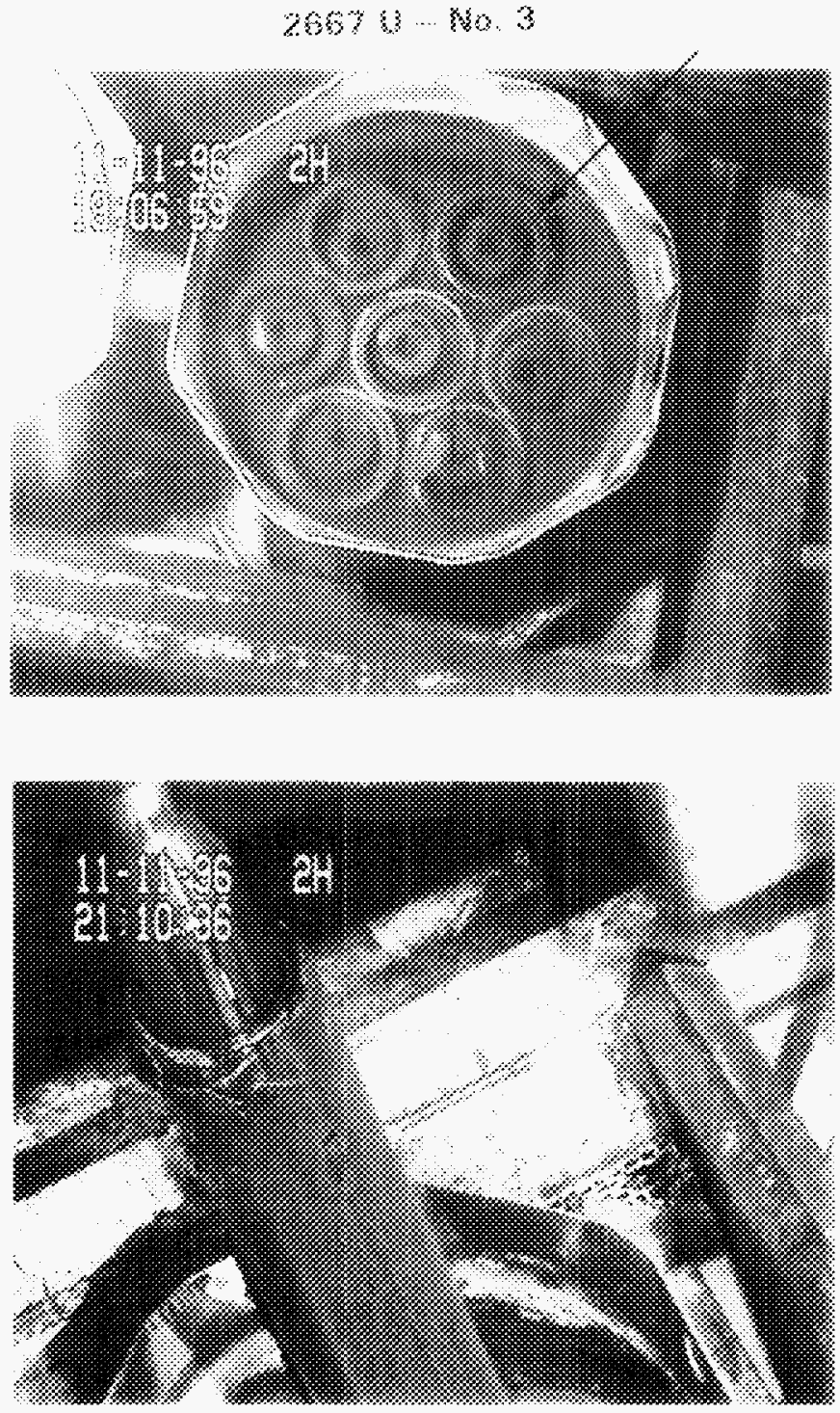
$3 \% 48 \ldots 30.4$
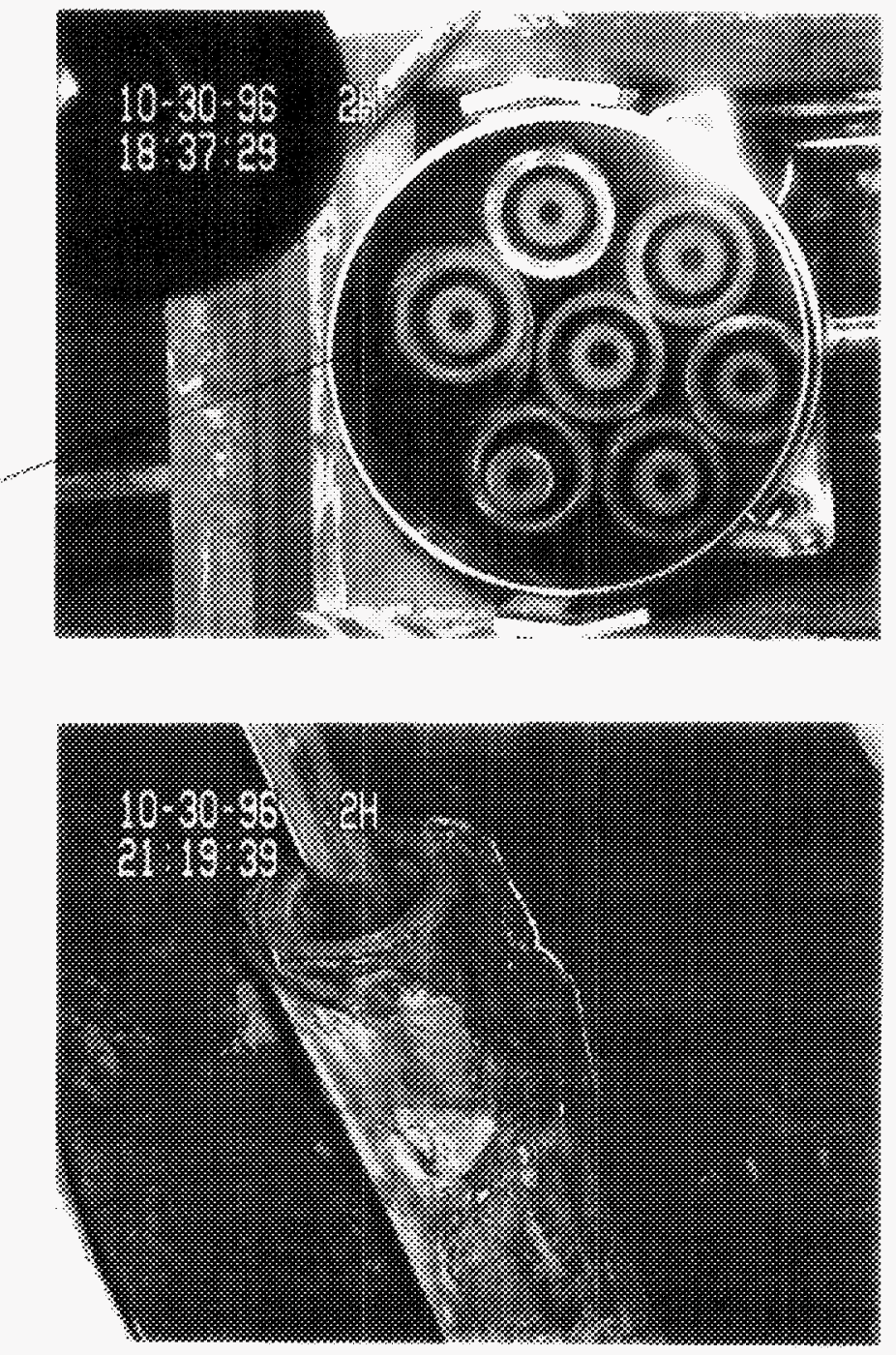
$8 \% 33 \ldots .30 .6$
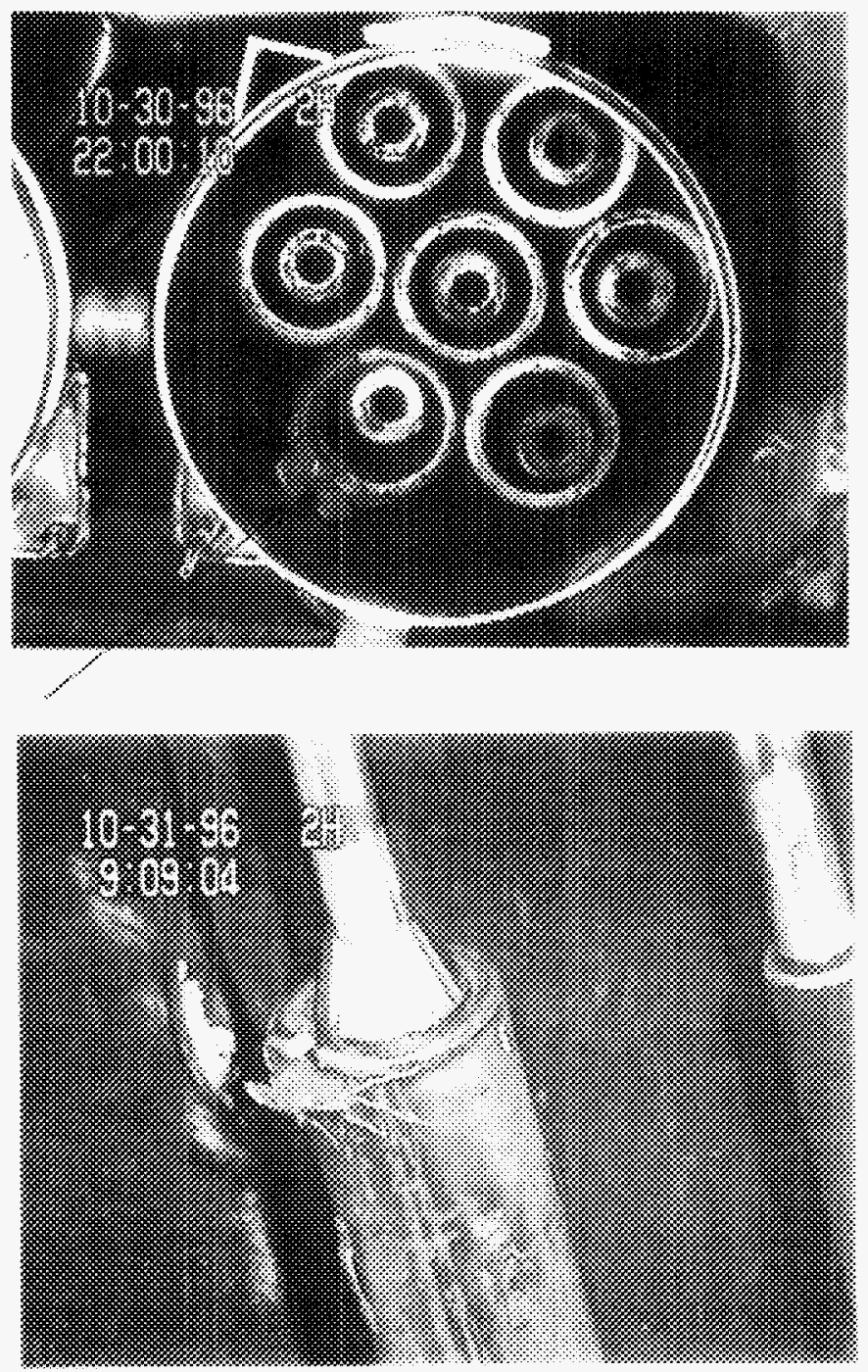


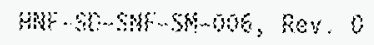

\section{$60 \% 3 \% .6$}
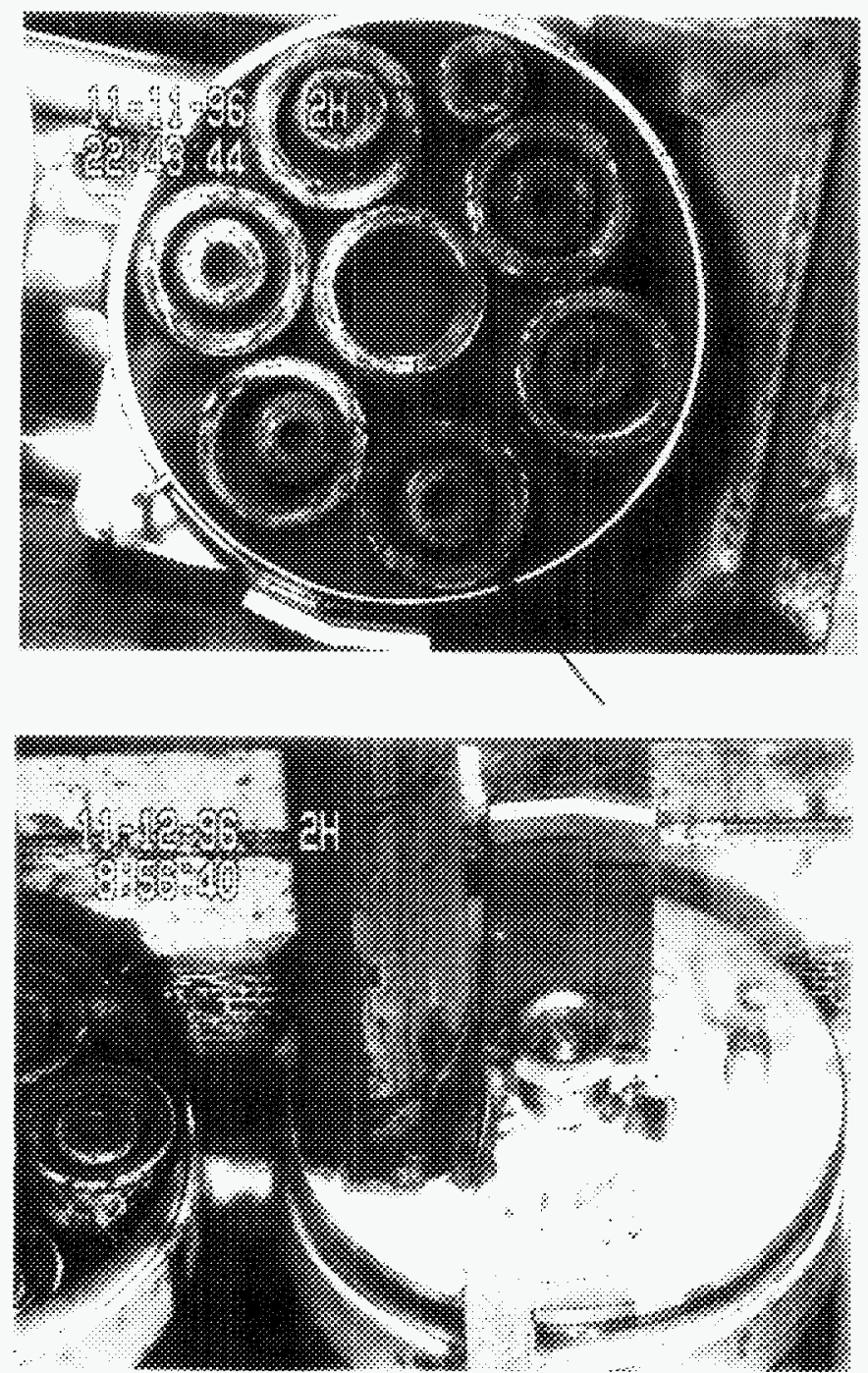

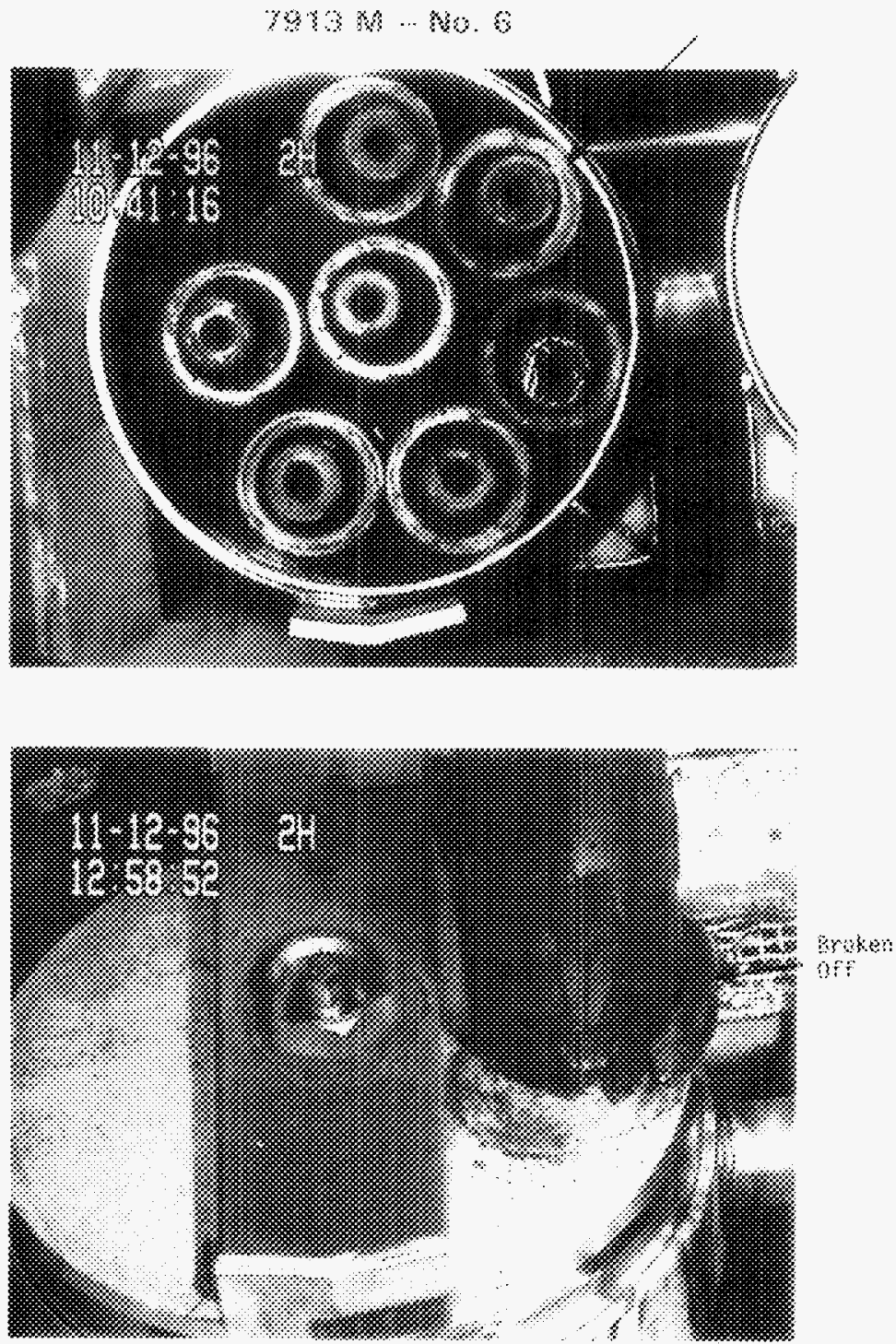


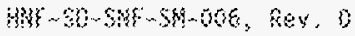

$5743\} \ldots$ Ko. 6
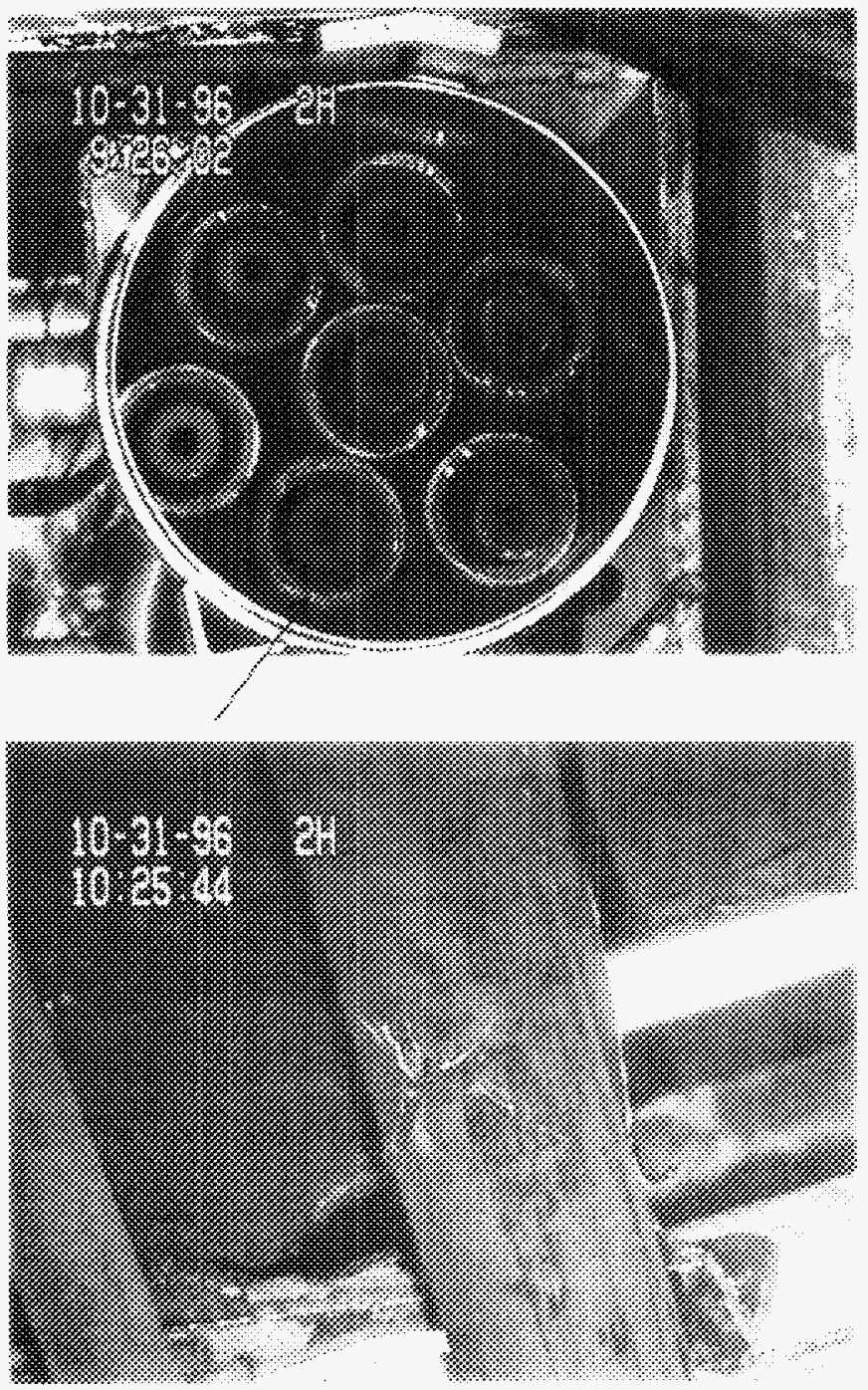
$7913: \ldots 80,4$
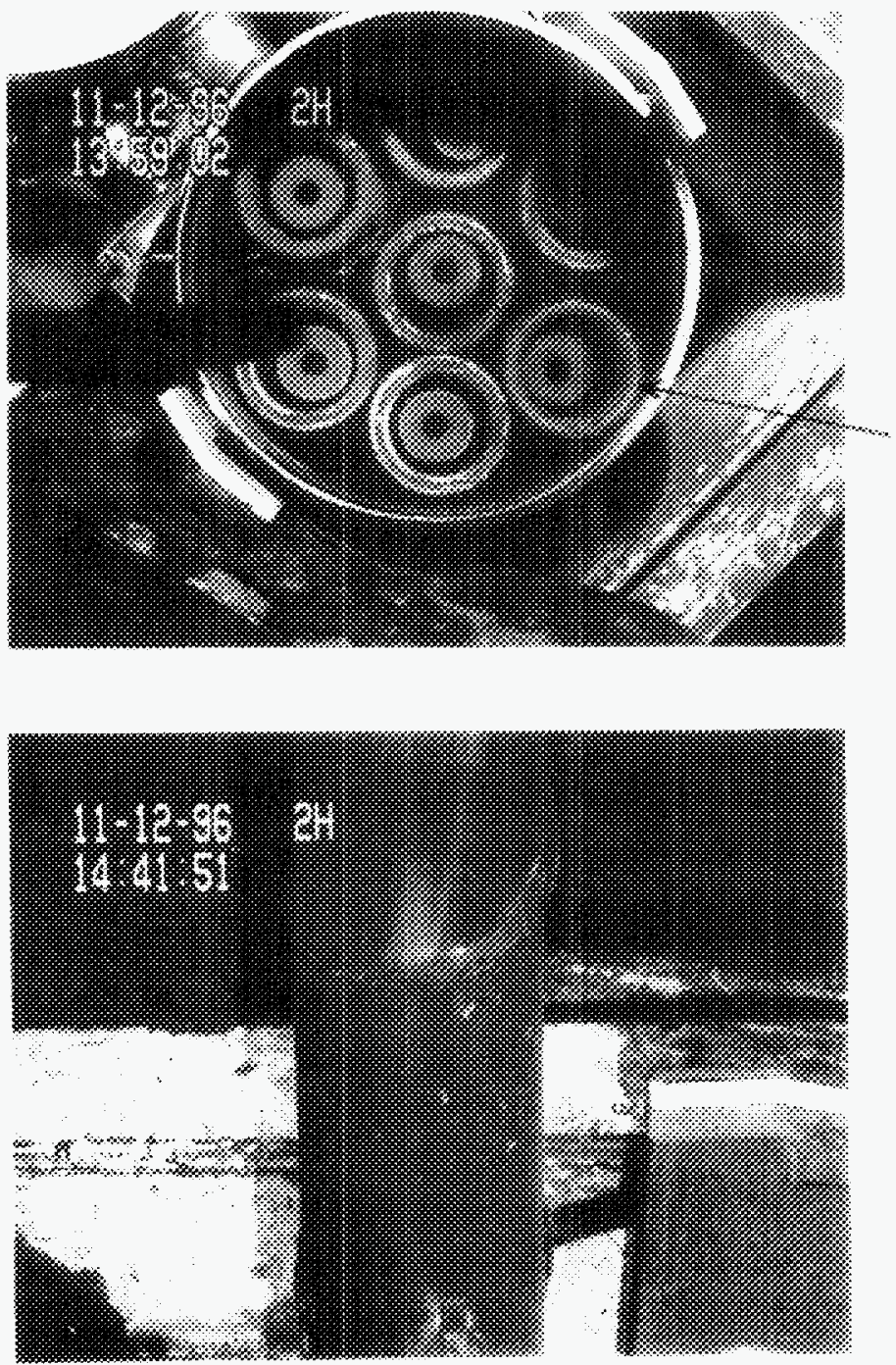


\section{DISTRIBUTION SHEET}

\begin{tabular}{|c|c|c|c|c|c|c|}
\hline To & \multirow{2}{*}{\multicolumn{3}{|c|}{$\begin{array}{l}\text { From } \\
\text { Spent Nuclear Fuel Evaluations } \\
2 T 650\end{array}$}} & \multicolumn{3}{|c|}{ Page 1 of 2} \\
\hline Distribution & & & & \multicolumn{3}{|c|}{ Date February 11, 1997} \\
\hline \multicolumn{4}{|l|}{ Project Title/Work Order } & \multicolumn{3}{|c|}{ EDT No. 617444} \\
\hline Spent Nuclear Fuel Pros & SD-SNF-SM-006, & ev. 0 & & \multicolumn{3}{|c|}{ ECN No. } \\
\hline \multicolumn{2}{|l|}{ Name } & $\begin{array}{l}\text { Text } \\
\text { With All } \\
\text { Attach. }\end{array}$ & \multicolumn{2}{|c|}{ Text Only } & $\begin{array}{l}\text { Attach./ } \\
\text { Appendix } \\
\text { Only }\end{array}$ & $\begin{array}{c}\text { EDT/ECN } \\
\text { Only }\end{array}$ \\
\hline
\end{tabular}

U.S. Department of Energy,

Richland Operations office

J. Shuen

$57-41 \quad X$

B\&w Protec Inc.

T. L. Welsh

T4-40 X

Fluor Daniel Northwest

M. Kummerer

G. A. Ritter

A3-34 $X$

$\mathrm{HO}-31 \quad \mathrm{X}$

Pacifjc Northwest National Laboratory

J. Abrefah

$\begin{array}{ll}\text { P7-27 } & X \\ \text { P7-27 } & X\end{array}$

S. C. Marschman

P7-27

\section{WSTRN}

N. R, Roe

$\mathrm{H} 0-40 \quad \mathrm{X}$

Duke Engineering and Services Hanford, Inc.

R. B. Baker

D. W. Bergmann

K. H. Bergsman

A. E. Bridges

L. D. Bruggeman

C. Defigh-Price

J. R. Gregory

F. G. Hudson

J. J. Jernberg

L. A. Lawrence

P. G. LeRoy

D. P. Maassen

P. J. MacFarlan

B. J. Makenas

C. R. Miska

K. R. Morris

R. P. Omberg

T. R. Pauly
$\mathrm{HO}-40 \quad \mathrm{X}$

R3-86 $X$

R3-48 $X$

HO- 40 X

HO-40 X

$X 3-79 \quad X$

$\times 3-72 \quad x$

R3-11 $X$

$\times 3-72 \quad X$

HO- 40 X

R3-15 $X$

HO- 40 X

$H 0-40 \quad x$

HO- $40 \quad X$

R3-86 $X$

$X 3-67 \quad x$

HO-40 $X$

$\times 3-85 \quad X$ 
DISTRIBUTION SHEET

\begin{tabular}{|c|c|c|c|c|c|c|}
\hline \multirow{2}{*}{$\begin{array}{l}\text { To } \\
\text { Distribution }\end{array}$} & \multirow{2}{*}{\multicolumn{3}{|c|}{$\begin{array}{l}\text { From } \\
\text { Spent Nuclear Fuel Evaluations } \\
\text { 2T650 }\end{array}$}} & \multicolumn{3}{|c|}{ Page 2 of 2} \\
\hline & & & & \multicolumn{3}{|c|}{ Date February 11, 1997} \\
\hline \multicolumn{4}{|l|}{ Project Title/Work Order } & \multicolumn{3}{|c|}{ EDT No. 617444} \\
\hline \multicolumn{4}{|c|}{ Spent Nuclear Fuel Project/HNF-SD-SNF-SM-006, Rev. 0} & \multicolumn{3}{|c|}{ ECN No. } \\
\hline Name & MSIN & $\begin{array}{l}\text { Text } \\
\text { With All } \\
\text { Attach. }\end{array}$ & Text & & $\begin{array}{l}\text { Attach./ } \\
\text { Appendix } \\
\text { Only }\end{array}$ & $\begin{array}{c}\text { EDT/ECN } \\
\text { Only }\end{array}$ \\
\hline
\end{tabular}

Duke Engineering and Services Hanford, Inc. (Continued)
K. L. Pearce
A. L. Pitner
R. W. Rasmussen
R3-48
HO- 40
R3-86
D. W. Siddoway
$\mathrm{X} 3-71$
J. A. Swenson
D. J. Trimble
SNF Project Files
Centrat Files
R3-11
$\mathrm{HO}-40$
R3-11
A3-88
$x$
$x$
$x$
$x$
$x$
$x$
$x$

Fluor Daniel Hanford, Inc.

E. W. Gerber

R3-11 X

Numatec Hanford Corporation

P. M. Bourlard

R3-86

F. W. Bradshaw

R3-85

$x$

$x$ 